\title{
Reptiles and Amphibians in Deep Canyons: The Big Jacks and Little Jacks Creek Drainages of Owyhee County, Idaho
}

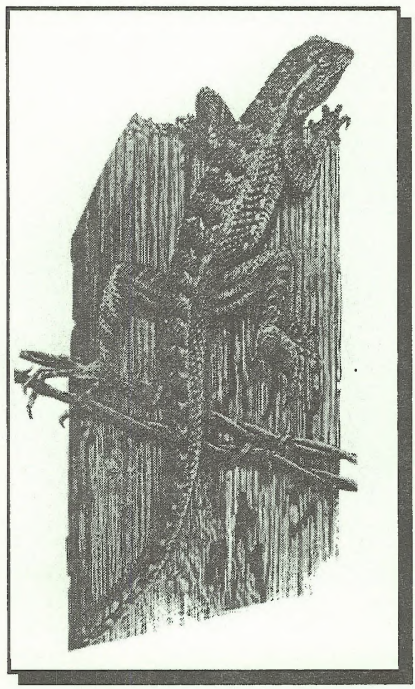


Reptiles and Amphibians in Deep Canyons:

The Big Jacks and Little Jacks Creek Drainages of Owyhee County, Idaho

\author{
A challenge cost-share agreement between \\ U.S. Bureau of Land Management and \\ Boise State University
}

December 13, 1996

Mark F. Gerber

Dr. James C. Munger

Aaron Ames

Jeff Stewart

Department of Biology, Boise State University, Boise, ID 83725

\title{
INTRODUCTION
}

Many of the reptiles and amphibians that live in southern Idaho can be found in the canyons of Owyhee county. However, of the few studies on reptiles and amphibians that have been conducted in Owyhee county (Diller 1981, Munger et al.1993, 1994, Beck 1994) none have been conducted in the deep canyon areas, such as Big Jacks and Little Jacks Creeks. These canyons, which can reach a depth of 400 meters, contain habitat suitable for many native reptiles and amphibians. However, it is presently unknown whether canyons represent a hot spot of herpetological diversity, and therefore are worthy of protection, or whether they are relatively depauperate, or are somewhere in between. This study will examine the diversity and abundance of reptiles and amphibians in deep canyons of Owyhee County, and should therefore provide a model for other similar areas in Southern Idaho. For the purposes of this report, all areas within the Big Jacks and Little Jacks Creeks drainages will be referred to as the "study area". The term "herp" is used in this report to collectively refer to reptiles and amphibians.

Of the species potentially found in the study area the Spotted Frog is listed by the U.S. Fish and Wildlife Service (USFWS) as a candidate species, the Idaho Department of Fish and Game (IDF\& $G$ ) as a species of special concem A (priority species), and by the Bureau of Land Management (BLM) as a sensitive species. The Northern Leopard Frog and the Mojave Black-collared Lizard are listed by the BLM as sensitive, and by DFFG as a species of special concern A. The Westem Toad is listed as an IDF\&G species of special concern C (undetermined status) and by the BLM as sensitive. The Western Ground Snake, and the Longnose Snake are listed by IDF\&G as species of special concern B (peripheral species).

This study was designed primarily as a survey to determine presence or absence of reptiles and amphibians of the deep canyons of the Big Jacks and Little Jacks creek drainages, and to provide baseline data for future studies. In addition, we collected data to determine movement patterns of snakes in the vicinity of Parker Trail on Big Jacks Creek. 


\section{METHODS AND MATERIALS}

Two methods of determining presence of herps were utilized; drift fences in conjunction with funnel traps, and visual encounter searches. These two methods were employed in four regimes: upland, rim, midslope/draws, and canyon bottom. The upland regime was not actively surveyed. Reptiles found in this regime were, therefore, incidental to the study, and included road sightings (both dead and alive) and reptiles that were seen while traveling within the study site. Rim sightings included animals encountered within $100 \mathrm{~m}$ of the rim, including those captured in drift fence arrays. Mid-slope sightings were characterized by draws, talus benches, and other suitable habitat that was below the cliff that defined the rim regime, but did not include talus that actually emerged onto the canyon bottom. Some areas of extremely steep canyon walls did not have a mid-slope regime. The canyon bottom was defined to include all sand bars, and talus or dirt that actually emerged onto the floor of the canyon.

Drift Fence installation and construction: Twenty seven drift fence arrays were placed in and near Big Jack's Creek Canyon, as shown in Fig. 1. Drift fences were constructed of two sections of material, either construction grade silt fence or strips of sheet metal flashing. Forty-three sections were constructed of steel flashing, each section used a piece $21^{\prime \prime}$ wide by $25^{\prime}$ long. The edge of each section was buried a minimum of six inches underground. On plateaus and windy slopes the bottom four inches was bent over before burying to keep the fence from blowing out of the ground. Steel fencing was installed in a trench that was no deeper than six inches and only wide enough to fit the steel in with the bottom four inches bent at a right angle to the fence (Fig. 2). Eight sections were constructed of silt fence, each section was twenty-five feet long by three feet wide with a two by two wooden post at each end and two spaced evenly between the end posts. The posts were driven into the ground at angles so they only contacted the fence at the top. Three foot long strip lathe was used to brace cloth that sagged due to uneven ground. Twenty-seven arrays were erected, 25 of which were comprised of 2 sections and the remainder of one twenty-five foot section of steel fence each. Of the twenty-seven arrays, eight were comprised of one section of steel fence and one section of silt fence, with the objective of determining whether or not there was a difference in effectiveness of each type of fence.

Funnel Traps: Funnel traps were constructed of aluminum window screen. A piece of screen was cut $24^{\prime \prime}$ on one side and $20^{\prime \prime}$ on the other. This piece was rolled into a cylinder $24^{\prime \prime}$ long and stapled together with household staples. One end of the cylinder was flattened, folded over, and clipped with clothespins. To make the funnel, a half circle was cut from the same material as the body and rolled into a funnel with a diameter of $22 \mathrm{~cm}$ at the widest point. The point end of the funnel was cut off to make a hole that was about $3 \mathrm{~cm}$ across. This funnel was inserted into the open end of the cylinder and stapled in place with the small hole inside the trap (Fig. 3). On each section of drift fence were placed four funnel traps, two on either side of each end of the fence. Each funnel trap was placed tightly against and parallel to the fence with the opening facing the center of the section of drift fence as in fig. 3. A dirt ramp was constructed into the opening of the funnel trap to keep reptiles from passing undemeath the trap and to help guide them into the trap. To give reptiles a place of refuge from the sun, a piece of cardboard 


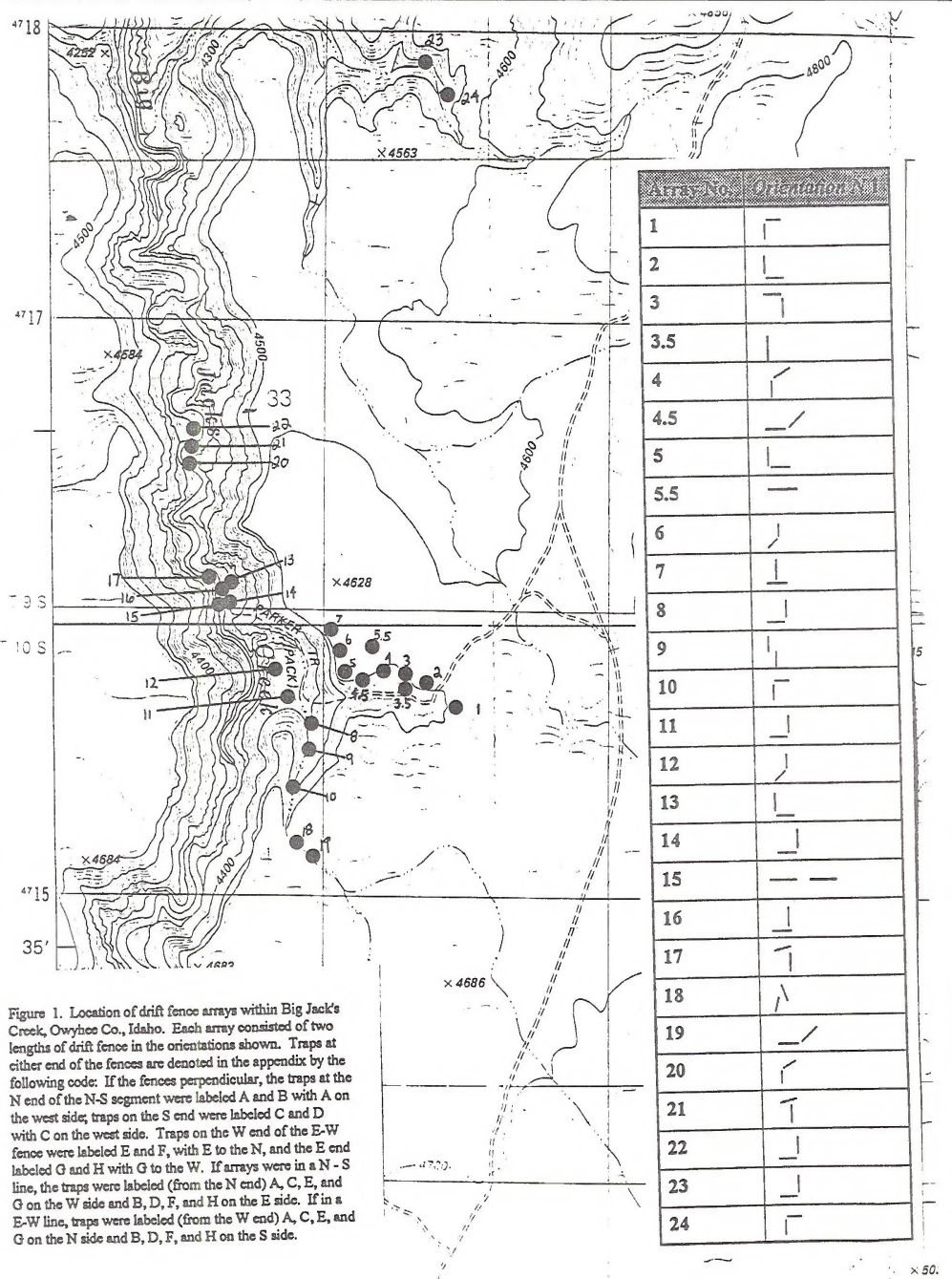




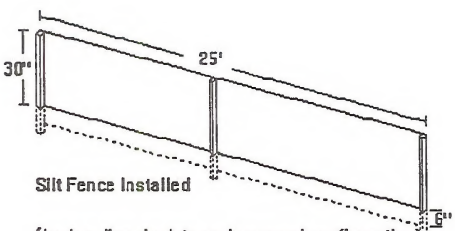

(broken line depicte underground configuratlon):

Figure 2a. Configuration of silt fence installation.

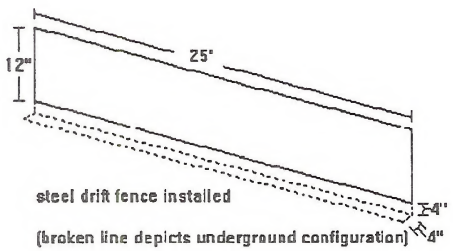

Figure $2 b$. Steel drift fence installation configuration. 


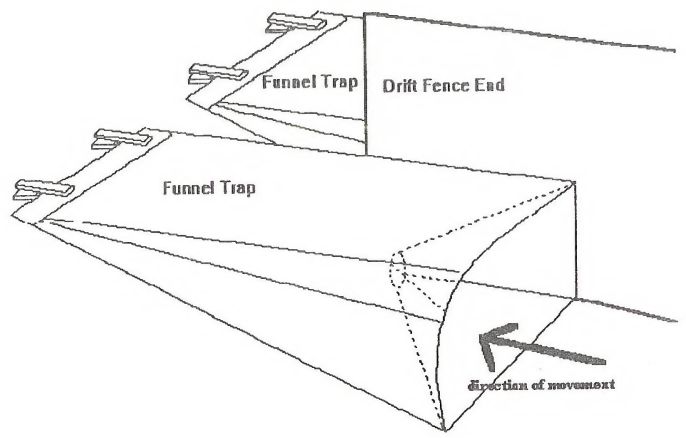

Figure 3. Position of traps on the drift fence end. 
was placed inside each trap and a shade of wood, cardboard, or branches was placed over the top.

Site Selection: Arrays were placed, when possible, at the junction of habitat types within each regime because of the greater likelihood of finding reptiles there that were moving between habitat types to forage. Arrays were placed in the regime defined for rim, mid-slope, and bottom of the canyon. Placement of the drift fence arrays was decided on site and on a per-array basis. Positioning of the arrays was based on practical placement with regards ease of installation, and presence of appropriate habitat.

Monitoring: The arrays were checked on a three day rotating basis to avoid casualties. Each trap was removed and thoroughly examined for the presence of animals. If a snake was caught in the trap, it was removed and sex, length, and species were recorded. Each snake within a $5 \mathrm{~km}$ radius of Parker Trail was marked with a unique number so it could be identified if recaptured. The marks were made on the ventral scales in the rear third of the snakes body. Scales were clipped to flank the number of unclipped scales for each digit of the marked number. For example, three clipped scales with three unclipped scales between the first two clipped scales and four between the second and third clipped scales would constitute number thirty-four. Lizards that were caught in traps were not marked, but all other data was recorded as for snakes. Animals other than reptiles or amphibians (e.g., birds and mammals) were released and no data was taken other than noting their presence in the trap.

Visual Searches: Visual searches consisted of selecting a section of canyon within the Big Jacks and Little Jacks Creeks drainages that was reasonably accessible, and hiking that section looking for reptiles. While performing visual searches, rocks and other surface debris were turned. All reptiles that were captured were sexed and sized, but not marked unless they were within $5 \mathrm{~km}$ of the drif fence arrays. Survey areas outside the trapping area were only surveyed once. All habitat that was disturbed when performing a visual search was replaced as close to its original condition as possible. When we encountered a skin from a reptile, it was identified to species, and recorded as a capture but note was made that it was a skin. We also noted the cloud cover, temperature in Celsius, time, location, and any distinguishing features of the reptiles encountered.

Visual searches of Big Jacks creek consisted of areas south of the Cottonwood/Big Jacks creek confluence three miles and north of the Cottonwood/Big Jacks creek confluence to the Wickahoney Creek confluence. We also conducted visual encounter searches for $1 / 4 \mathrm{mi}$ north and south of where Big Jacks creek crosses Battle creek road. We surveyed Cottonwood creek from Cottonwood springs to the Big Jacks creek confluence. We surveyed a one mile section of Big Jacks creek, five miles south of the Big Jacks/Little Jacks creek confluence.

The confluence of Big Jacks/Little Jacks creek was surveyed extensively \&s well as tributaries in the vicinity and surrounding hills. This area was visited on three occasions because of the frequency that we passed through on our way to other sections of the creeks. The bench above the confluence was searched as well as Rattlesnake creek which drains the bench into Little Jacks creek.

Little Jacks creek was surveyed in fewer places because of limited access points. We surveyed a one mile section of the creek near OX reservoir and approximately two miles south of 
the confluence with Big Jacks creek. We also surveyed approximately one mile of Little Jacks creek on either side of the Rattlesnake creek confluence, and a one mile section beginning approximately two miles south of the Rattlesnake creek confluence.

In addition to the main creeks we conducted visual encounter surveys in other areas that contained apparently good habitat for herps. Most of these areas fell along roads that travelled parallel to the main creeks. The areas that we visited on these occasions were: Hole in Rock, Big Horse Basin Gap, Rattlesnake Gulch, Wickahoney, Whites Reservoir, Mud Flat Road and Shoofly Cutoff Road.

\section{RESULTS}

A total of 623 reptiles were encountered during the course of the study. Two hundred eighty-five reptiles were caught in the rim regime, 53 were caught mid-slope, and 116 were caught on the canyon bottom. The remaining 169 reptiles were incidental to the study.

Snakes:

Six species of snakes were found in the canyons of the study area: Gopher Snakes, Western Whipsnakes, Western Rattlesnakes, Night Snakes, Western Terrestrial Gartersnakes, and Western Racers. An additional two species that were found incidental to the study: Western Ground Snakes, and Longnose Snakes. Snakes were found in approximately the same numbers on the rim as in the canyon bottoms. Only a small percent of the snakes found were found mid-slope (Fig. 4). Snakes amounted to about one third of all reptiles caught on the rim, as did snakes caught mid-slope. Snakes accounted for about $80 \%$ of all reptiles caught on the canyon bottom. Eighty-nine snakes were marked within $5 \mathrm{~km}$ of Parker Trail. A total recapture count of each species appears in Fig. 5.

Lizards:

Seven species of lizards were found in the canyons surveyed; Side-blotched lizards, Western Fence lizards, Desert Horned lizards, Short Homed lizards, Longnose Leopard lizards, Western Whiptails, and Western Skinks. One additional species was found incidental to the study, the Mojave Black-collared lizard. Lizards comprised about $70 \%$ of the reptiles found on the rim, and two-thirds of those reptiles found mid-slope. Lizards accounted for only about $20 \%$ of the reptiles found on the canyon bottom; lizards were found almost strictly on the rim (Fig. 6).

Amphibians:

There was only one species of amphibian found in the study area, Pacific Tree Frog, which was identified by Jim Munger, by call. All others were incidental to the study. This frog accounted for one percent of all herps found on the canyon bottom. 
Herp Survey Data 1995

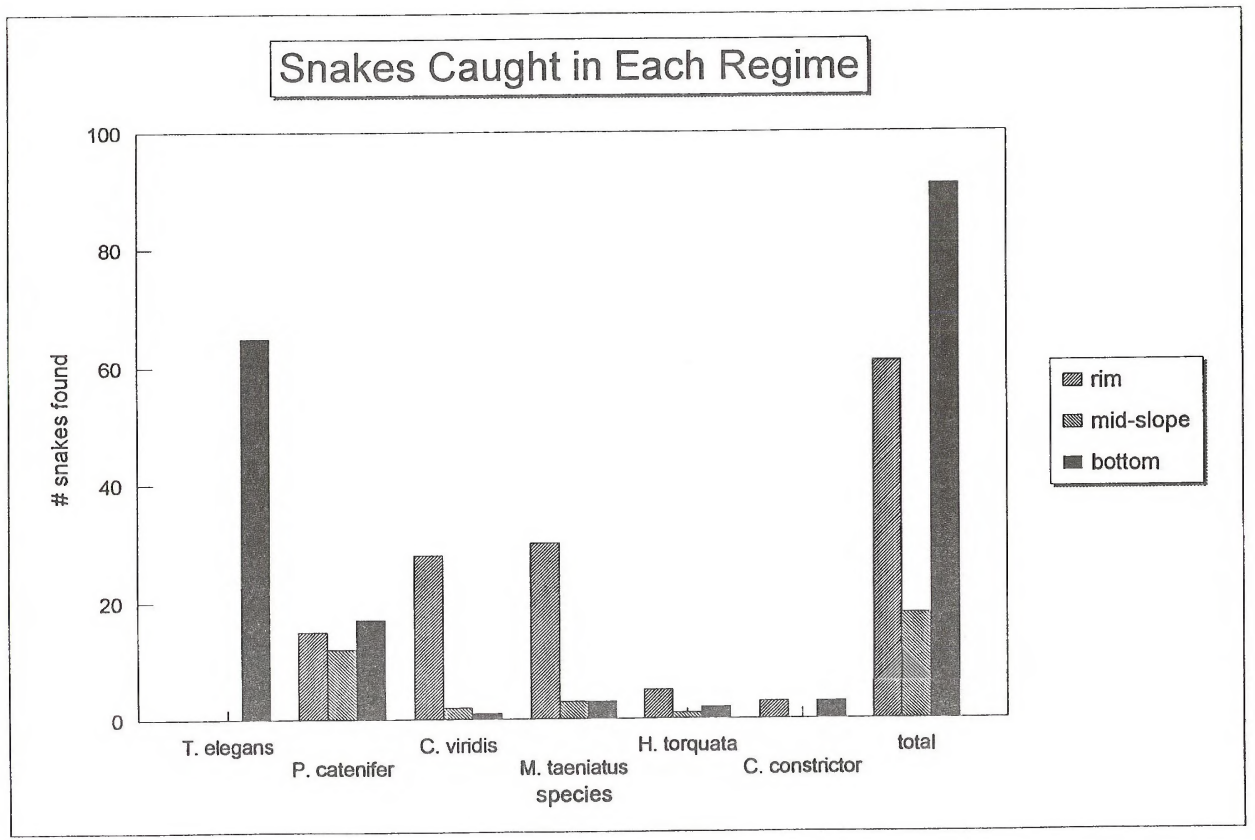

Figure 4. 
Herp Survey Data 1995

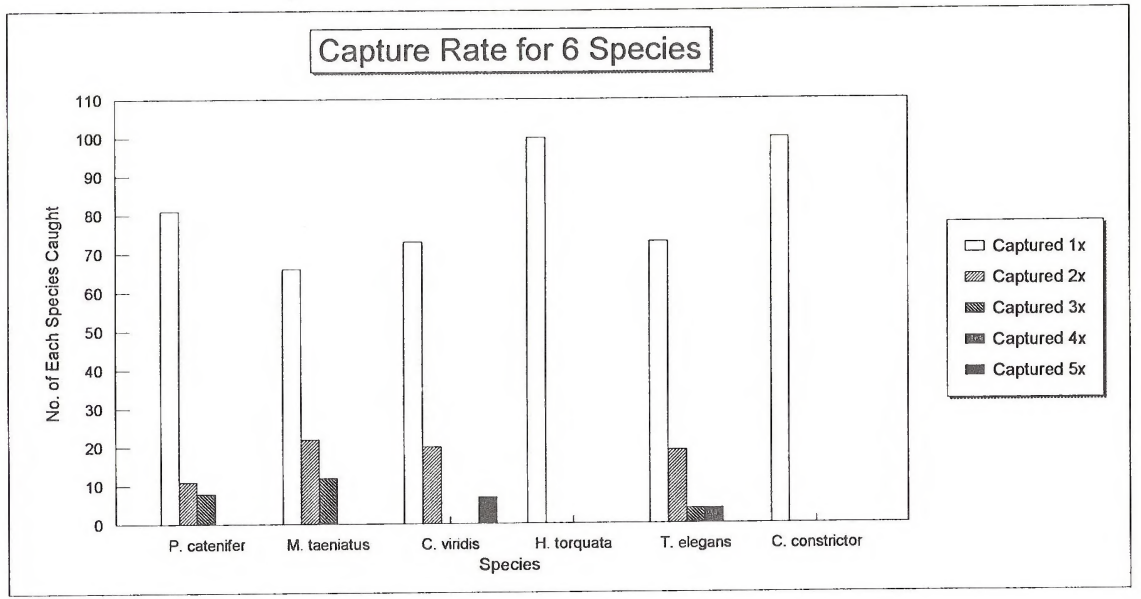

Figure 5. 
Herp Survey Data 1995

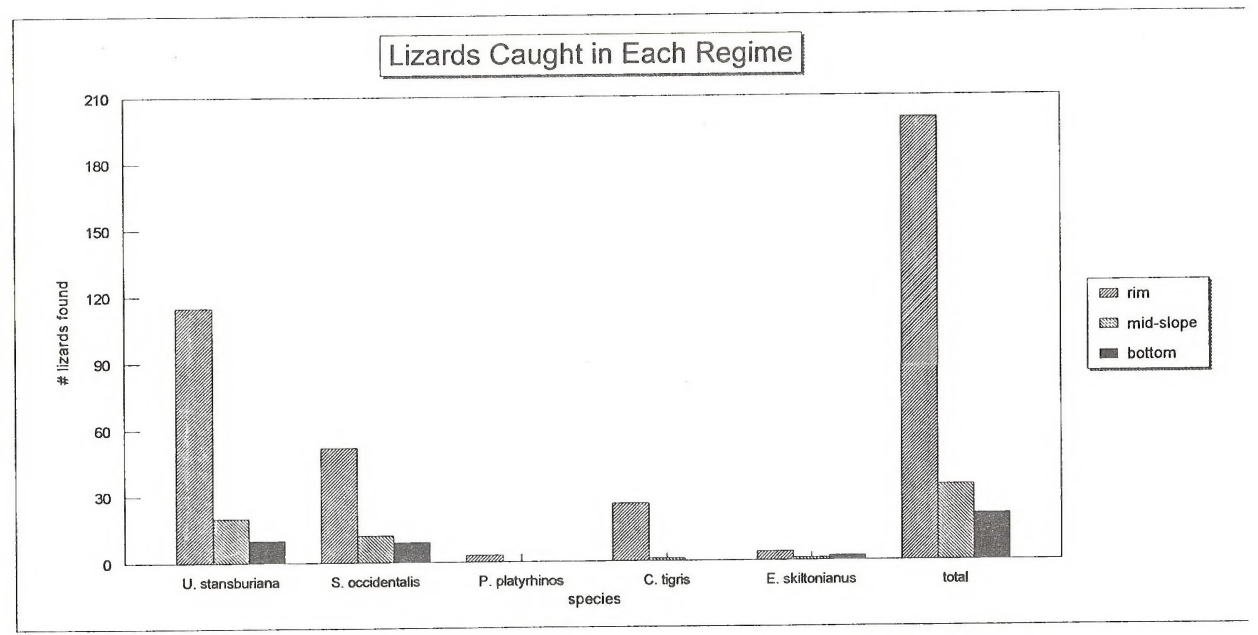

Figure 6 
Visual Encounter Searches:

In addition to the data obtained from trapping, we found some notable herps during our visual encounter searches. On July 3, we stopped at Big Horse Basin Gap to survey primarily for Crotaphytus bicinctores. We found only one C. bicinctores, but in the wash that emerges from. the gap, we found an unusually high number of Sonora semiannulata skins and living specimens. We also found one Hypsiglena torquata. All herps except the $C$. bicinctores were found by turning rocks. We retumed to this site on Sept. 9, and found two C. bincinctores juveniles and no fresh $S$. semianmulata skins nor live specimens. We found $C$. bicinctores on hot sunny days in rocky areas north of the main bench. We found a C. bicinctores population at Big Jacks Creek where the pipeline and the creek meet. We found them consistently for about $.5 \mathrm{mi}$ to the east of Big Jacks creek and for about $1.5 \mathrm{mi}$ to the west. We found a population on the Oreana-Triangle road just before climbing the bench.

\section{DISCUSSION}

Based on their geographic ranges (Nussbaum et al. 1985 and Stebbins 1986) the following reptiles and amphibians potentially occur in the study area; those marked with an asterisk were found during the course of this study.

Snakes:

*Westem Rattlesnake (Crotalus viridis)

* Great Basin Gopher Snake (Pituophis catenifer)

*Westem Yellow-bellied Racer (Coluber constrictor)

*Western Striped Whipsnake (Masticophis taeniatus)

*Western Ground Snake (Sonora semiannulata)

*Night Snake (Hypsiglena torquata)

*Westem Longnose Snake (Rhinocheilus lecontei)

*Western Terrestrial Garter Snake (Thamnophis elegans)

Common Garter Snake (Thamnophis sirtalis)

Rubber Boa (Charina bottae)

Lizards:

*Longnose Leopard Lizard (Gambelia wislizenii)

*Western Whiptail (Cnemidophorus tigris)

*Desert Horned Lizard (Phrynosoma platyrhinos)

*Short Horned Lizard (Phrynosoma douglassi)

* Side-blotched Lizard (Uta stansburiana)

*Western Fence Lizard (Sceloporus occidentalis)

*Sagebrush Lizard (Sceloporus graciosus)

*Mojave Black-collared Lizard (Crotaphytus bicinctores)

*Westem Skink (Eumeces skiltonianus) 
Amphibians:

* Pacific Treefrog (Pseudacris regilla)

Spotted Frog (Rana pretiosa)

Northern Leopard Frog (Rana pipiens)

Westem Toad (Bufo boreas)

Woodhouse's Toad (Bufo woodhousei)

Great Basin Spadefoot Toad (Spea intermontamus)

Reptiles and amphibians potentially found in the study area represent $69 \%$ of the total species of reptiles and amphibians in Idaho. Additionally, Long-toed Salamanders (Ambystoma macrodactylum) could possibly be found in the study area because the salamander's range borders the study area, and habitat within the study area is similar to the preferred habitat of the salamander. As marked by an asterisk above, we found 17 of 19 potential reptile species during the course of the study.

We found reptiles in all three of the regimes that we surveyed: canyon bottom, mid-slope, and rim. The rim and canyon bottom regimes contained the most reptiles; relatively few were seen in the mid-slope regime, especially during the hottest part of the summer (Fig. 7).

Big Jacks Creek was surveyed using mainly standard drift fence arrays. The drif fence arrays were of two types, steel flashing and silt fence. Silt fence was set up to determine its effectiveness. Insufficient data was obtained to determine to what extent silt fence is effective. Lizards were seen climbing on the silt fence itself. Snakes have been observed using the upright posts to climb over the fence if the posts are placed too close to the fence (M. Gerber pers. obs.). We angled the uprights to eliminate the latter problem. Movement by reptiles in the trapping area was negligible, with very few being recaptured more than $50 \mathrm{~m}$ from the original site of capture. We did capture an unusually high number of rattlesnakes in one array on the rim in the spring, but by mid summer we could only find one. This could be the result of a den in the vicinity of the array.

Visual searches did not appear to be effective during this study. We found a limited number of species of reptiles using this method. The areas that were surveyed were very rocky, and although rocks were turned, there were many deep talus runs that enabled snakes to retreat far below the surface, thus limiting the habitat that was practical to survey using this method. Except for one Western Groundsnake population, all reptiles that were seen were active foraging, diurnal species such as Gopher snakes, Racers, Whipsnakes, and Western Terrestrial Garter snakes. We occasionally encountered Western Rattlesnakes, which are usually crepuscular but were found frequently during mid morning hours. Representatives of all nine species of lizards found in this study were found during visual searches. Most of the lizards were found on the canyon rim while lizards on the canyon bottom were second in abundance. Mojave Black-collared lizards and Longnose Leopard lizard were recorded as incidentals. 
Herp Survey Data 1995

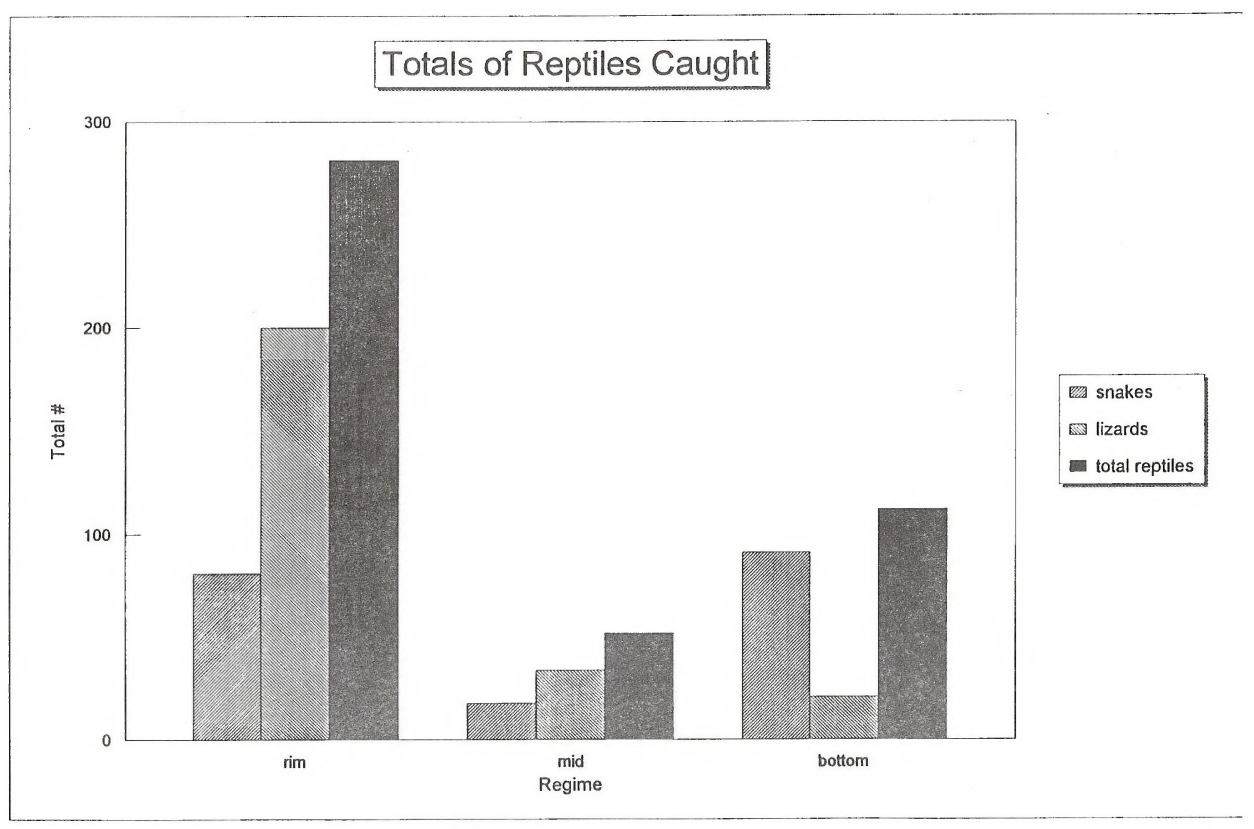

Figure 7 


\section{SUMMARY}

Sixteen species of reptiles and amphibians were found in this study, of which 13 were associated with the deep canyons. Canyon rims and canyon bottoms were utilized by reptiles the most. Western Terrestrial Garter Snakes were found strictly on the bottoms, and Western Rattlesnakes were found primarily on the rim. Gopher Snakes, Racers, and Striped Whipsnakes were found to be relatively abundant in all three regimes surveyed. There appeared to be little or no vertical movement of reptiles between regimes. These deep canyons are important areas in the desert for reptiles because they provide apparently suitable cover and forage for most of Idaho's reptiles. Most of the waterways in these deep canyons are perennial and provide apparently good habitat for reptile and amphibian species.

\section{LITERATURE CITED}

Beck, J. M. and C. R. Peterson. 1995. Movements and habitat selection of the longnose snake (Rhinocheilus lecontei) in southwestern Idaho. Idaho Bureau of Land Management, Technical Bulletin 95-18.

Diller, L. V. and D. R. Johnson. 1982. Ecology of reptiles in the Snake River Birds of Prey Area. Report to the Bureau of Land Management.

Munger, J., L. Heberger, D. Logan, W. Peterson, L. Mealy, and M. Caughlin. 1994. A survey of the herpetofauna of the Bruneau Resource Area, with focus on the Spotted Frog, Rana pretiosa. Idaho Bureau of Land Management Technical Bulletin No. 94-7.

Munger, J. C., M. Gerber, M. Carroll, K. Madrid, T. Bert. 1995. Herpetofauna of Bruneau and Owyhee Resource Areas, Boise District, with focus on the Spotted Frog, Rana pretiosa: a second year. Idaho Bureau of Land Management Technical Bulletin No. 96-1.

Nussbaum, R., E. Brodie, Jr., and R. Storm. 1983. Amphibians and reptiles of the Pacific northwest. University Press of Idaho, Moscow, Idaho.

Stebbins, R. C. 1985. A field guide to western reptiles and amphibians. Houghton Mifflin Co., Boston. 


\begin{tabular}{|c|c|c|c|c|c|}
\hline ppendes & apresein & gontuaton tor sumt & for of 195 & teris & \\
\hline$\quad 7$ & Fepimies: & bottom = bottom: & d canyon ins $\Rightarrow$ & pidslope & $r=n$ \\
\hline & JYML ar & de Eevelione were. & balest from Use & ST.5.to & gogra \\
\hline Date & Time & Genus & Spscias & regime & $\begin{array}{c}\text { Mark } \\
\text { 䊅 }\end{array}$ \\
\hline Amphibians & & & & & \\
\hline $05 / 18 / 95$ & 900 & Pseudacris & regilla & 1 & no \\
\hline $05 / 19 / 95$ & 1440 & Pseudacris & regilla & b & no \\
\hline $06 / 19 / 55$ & 1567 & Pseudacns & regilla & i & no \\
\hline $06 / 19 / 95$ & 1557 & Pseudacne & regilla & $i$ & no \\
\hline $05 / 18 / 85$ & 1003 & Psoudache & regilia & 1 & no \\
\hline Lizards & & & & & \\
\hline $07 / 103 / 95$ & 1744 & Cnemidophons & tigns & I & no \\
\hline $07 / 03 / 95$ & 1437 & Cnemidophons & tong & 1 & no \\
\hline $07 / 03 / 95$ & 1731 & Cnemidophoris & tigris & $r$ & no \\
\hline $07 / 03 / 95$ & 1202 & Cnemidophone & tigns & 1 & no \\
\hline $07 / 103 / 95$ & 1156 & Cnemidophons & tignis & 1 & no \\
\hline $07 / 03 / 95$ & 1030 & Cnemidophoris & tigns & $i$ & no \\
\hline $07 / 05 / 55$ & 2027 & Cnemidophons & tipns & $r$ & no \\
\hline $07 / 08 / 95$ & 929 & Cnemidophoris & tigris & $\mathrm{i}$ & no \\
\hline $07 / 08 / 55$ & 1058 & Cnemidophons & tigns & i & no \\
\hline 07108195 & 1052 & Cnemidophons & tignis & $P$ & no \\
\hline $07 / 12 / 95$ & 1318 & Cnemidophoris & tigns & $t$ & no \\
\hline $07 / 12 / 95$ & 1315 & Cnemidophons & tigns & i & no \\
\hline $07 / 23 / 95$ & 1050 & Cnemidophors & tigris & $i$ & no \\
\hline $07 / 29 / 95$ & 832 & Cnemidophoris & tigns & $r$ & no \\
\hline $07 / 29 / 95$ & 638 & Cnemidophoris & tigns & I & no \\
\hline $08 / 29 / 95$ & 811 & Cnemidophors & tigns & $r$ & no \\
\hline $05 / 25 / 35$ & 1304 & Cnemidophorus & tignis & $r$ & no \\
\hline $05 / 25 / 95$ & 1337 & Cnemidophorus & tigns & $m$ & no \\
\hline $05 / 27 / 95$ & 1159 & Cnemidophorus & tipris & 1 & no \\
\hline $05 / 29 / 95$ & 1718 & Cnemidophorus & tipris & I & no \\
\hline $05 / 30 / 95$ & 1157 & Cnemidophorus & tigris & I & no \\
\hline $06 / 12 / 95$ & 1300 & Cnemidophorus & tigns & 1 & no \\
\hline $06 / 12 / 95$ & 1305 & Cinemidophorus & tons & $i$ & no \\
\hline $06 / 12 / 95$ & 1153 & Cnemidophorus & tigris & $i$ & no \\
\hline $06 / 12 / 95$ & 1257 & Cnemidophorus & tigns & $i$ & no \\
\hline $06 \sqrt{26195}$ & 1242 & Cnemidophorus & tigris & $r$ & no \\
\hline $06 \sqrt{26195}$ & 928 & Cnemidophorus & tigris & $i$ & no \\
\hline $06 \sqrt{26 / 95}$ & 1222 & Cnemidophorus & tigris & 1 & no \\
\hline $05 / 26 / 95$ & 11242 & Cnemidophorus & tipris & 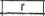 & no \\
\hline $06 \sqrt{26 / 95}$ & 1222 & Cnemidophorus & tigns & $r$ & no. \\
\hline $06 / 26195$ & 1221 & Cnemidophorus & thens & I & no \\
\hline $06 / 28 / 95$ & 1312 & Cnemidophorus & tigns & $r$ & no \\
\hline $06 / 28 / 95$ & 1157 & Cnemidophorus & tipns & I & no \\
\hline $06 / 29 / 95$ & 938 & Cnemidophorus & tigns & 1 & no \\
\hline $06 / 29 / 95$ & 1221 & Cnemidophorus & tigris & $i$ & no \\
\hline $06 / 29 / 95$ & 1221 & Cnemidophorus & tigns & $i$ & no \\
\hline $07 / 01 / 95$ & 1845 & Cnemidophorus & Iigns & i & no \\
\hline $07 / 101 / 95$ & 1029 & Cnemidophorus & tignes & 1 & no \\
\hline $07 / 15 / 95$ & 1330 & Cnemidophorts & tigns & $i$ & no \\
\hline $07 / 15 / 95$ & 1305 & Cnemidoghorus & tions & $i$ & no \\
\hline $07198 / 95$ & 1140 & Cnemidophorus & tigns & 1 & no \\
\hline $07 / 18 / 85$ & 1150 & Crnemidophorus & tigns & i & no. \\
\hline$0 6 \longdiv { 1 2 / 9 5 }$ & 1448 & Crotaphytus & bicinctores & i & no \\
\hline $0612 / 95$ & 1520 & Crolaphytus & bicinctores & 1 & no \\
\hline $06 / 12 / 95$ & 1520 & Crotaphytus & bicinctores & $i$ & no \\
\hline $06 / 12 / 95$ & 1520 & Crotaphylus & bicinctores & 1 & no \\
\hline $06 / 12 / 35$ & 1257 & Crotaphytus & bicinctores & 1 & no \\
\hline $08 / 12 / 95$ & 1257 & Crotaphytus & bicinctores & $\mathrm{i}$ & no \\
\hline $06 / 12 / 95$ & 1300 & Crotaphytus & bicinctores & I & no \\
\hline $06 / 12 / 95$ & 1511 & Crotaphytus & bicinctores & $i$ & no \\
\hline $06 / 12 / 95$ & 1519 & Crotaphytus & bicinctores & $i$ & no \\
\hline $06 / 12 / 95$ & 1511 & Crotaphytus & bicinctores & i & no \\
\hline $06 / 12 / 95$ & 1514 & Crotaphytus & bicinctores & 1 & no \\
\hline $06 / 12 / 95$ & 1511 & Crotaphytus & bicinctores & i & no \\
\hline $06 / 12 / 95$ & 1520 & Crolaphytus & bicinctores & i & no \\
\hline $06 / 12 / 95$ & 1448 & Crolaphytus & bicinctores & $\mathrm{i}$ & no \\
\hline $06 \sqrt{29 / 95}$ & 1210 & Crotaphytus & bicinctores & 1 & no \\
\hline $06 \sqrt{29 / 95}$ & 1213 & Crotaphytus & bicinctores & $i$ & no \\
\hline $06 / 29 / 95$ & 1147 & Crotaphutus & bicinctores & $i$ & no \\
\hline $06 \sqrt{29 / 95}$ & 1147 & Crotaphytus & bicinctores & $\mathrm{i}$ & no \\
\hline $06 \sqrt{29 / 95}$ & 1215 & Crotaphylus & bicinctores & i & no \\
\hline 07/03/95 & 1149 & Crotaphytus & bicinctores & 1 & no \\
\hline $07 / 22 / 95$ & 1435 & Crotaphytus & bicinctores & 1 & no \\
\hline $09 / 09 / 95$ & 1330 & Crotaphylua & bicinctores & $i$ & no \\
\hline $09 / 09 / 95$ & 1330 & Crotaphintus & bicinctores & i & no \\
\hline $05 / 20 / 95$ & 1930 & Eumeces & skiltonianus & 1 & no \\
\hline $05 / 30 / 85$ & 816 & Eumeces & skitonianus & $r$ & no \\
\hline $06 / 01 / 95$ & 2041 & Eumeces & skiltonianus & $b$ & no \\
\hline $06 / 11 / 95$ & 1053 & Eumeces & skiltonianus & b & no \\
\hline $06 \sqrt{28195}$ & 1620 & Eumeces & skiltonianus & $m$ & no \\
\hline $07 / 101 / 85$ & 1900 & Eumeces & skittonianus & $r$ & no \\
\hline $07 / 15 / 95$ & 1305 & Eumeces & skitonianus & $t$ & no \\
\hline $06 / 06 / 95$ & 1717 & Gambelia & wisilzenii & 1 & no \\
\hline $06 / 06 / 95$ & 1707 & Gambelia & wisizeni & I & no \\
\hline $08 / 12195$ & 1232 & Gambelia & wistizenil & $i$ & no \\
\hline$0 8 \longdiv { 2 9 1 9 5 }$ & 954 & Gambelia & wislizenii & i & no \\
\hline $07103 / 95$ & 1033 & Gambelia & islizenil & $r$ & no \\
\hline $07 / 18 / 95$ & 1207 & Gambelia & wisluzenii & $i$ & no \\
\hline $07 / 18 / 95$ & 920 & Gambelia & wislizenu & $m$ & no \\
\hline $07 / 22 / 95$ & 1400 & Gambelie & wsizenii & i & no \\
\hline $07 / 24 / 95$ & 1917 & Phrynosoma & douglassi & 1 & no \\
\hline $06 / 18 / 93$ & 1443 & Phrynosoma & douglassil & 1 & no \\
\hline
\end{tabular}




\begin{tabular}{|c|c|c|c|c|}
\hline $00 \mathrm{n}$ & $0068 \angle 9$ & $0892+\angle P$ & LOJS $3 p$ Y SOL 1 & \\
\hline 0095 & oseess & $09 \angle S L \angle S$ & 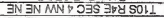 & S'L \\
\hline 0097 & 08108 & $O L L S L L Y$ & 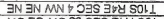 & $\varepsilon$ \\
\hline Colb & 029299 & $0 Z 191<7$ & 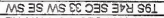 & $\overline{c L}$ \\
\hline 085 & OLBZSG & $00 \angle 91 . \angle 9$ & 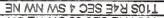 & $L$ \\
\hline $09+4$ & C8EEs: & $08 \angle L L \angle 7$ & 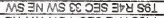 & $L$ \\
\hline OZNS & $0 \angle 6299$ & $0 Z$ CSILY & $\Xi N$ MN MS $>0 \Xi S \exists \square Y$ SOL 1 & 9 \\
\hline 0097 & OSDEBS & Orogl:Lt & 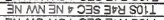 & SL \\
\hline 0297 & OZEZBG & $0 \angle L 91 \angle D$ & EN MS MS $>03 S$ JYY SOL1 & SL \\
\hline $029 y$ & 028289 & OLLGLLD & 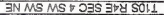 & 8 \\
\hline 9998 & 005949 & Cavo\&Lb & MS MN $3 S \rightarrow 1$ OAS $\exists E C$ S81 & $L$ \\
\hline 0095 & orocos & $0969 . \angle 7$ & 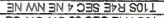 & 8 \\
\hline $009 \%$ & ONOESS & 0.6 .9 .47 & 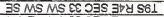 & CंL \\
\hline 0960 & $08 \mathrm{geg}$ & $0 \Omega \angle \angle L P B$ & MS $3 N M S$ CE OSS 374 S 81 & \\
\hline 0915 & OlLZOS & $0909, \angle 7$ & IN MW NWN WOES $\exists Y 4$ SOLL 1 & 8 \\
\hline $00 \%$ & $08528 \mathrm{C}$ & 0299147 & ZNMS $\exists N$ \& $2 \exists S$ JPY SOLI & SूL \\
\hline $08 \angle Z$ & $0 Z 8189$ & OZEEELY & MN MS MS S J JS $\exists \square Y$ S 1 & $L$ \\
\hline $0 \rightarrow 28$ & $0, Z 999$ & OISZELL & 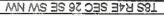 & 2 \\
\hline $092 \varepsilon$ & DQBLPC & $000 Z \varepsilon \angle Y$ & MN MS $3 S$ gZ $59 S$ JPO S81 & 6 \\
\hline $0 \rightarrow Z \varepsilon$ & OZZSOS & OATEZEL & 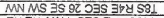 & 8 \\
\hline $\cos \theta$ & $0 \cos s$ & OSLCILD & 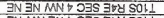 & 8 \\
\hline 0095 & 850689 & $80691 . \angle 7$ & 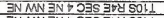 & $L$ \\
\hline oght & SLOCOS & $S L Q S, \angle P$ & $\exists N M N=\exists N \Rightarrow 03 S$ SPY SOLL & SL \\
\hline 0095 & OCOEgG & $07891 / 47$ & 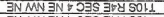 & \\
\hline $\cos 9$ & otoesc & S50 $91 \angle \nabla$ & JN MN $\equiv N>03 S$ S $\$ P Y$ SOL 1 & SL \\
\hline OSG & cogess & $00 \angle 9, \angle D$ & MN $\Xi S$ JS WE J $3 S$ JPY S61 & 8 \\
\hline $060^{\circ}$ & ogszas & $00996 \angle 7$ & MS JN MN EC OSS 374 S61 & SL \\
\hline ow & 008299 & S9E:LVID & 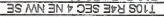 & $\frac{\pi}{2}$ \\
\hline Dost & 998295 & S9991LV & $\exists N$ MN MS $>0 \exists S$ JVY SOLL & EL \\
\hline $08 \mathrm{cos}$ & 59825 & $9895: \angle b$ & $\exists N M N$ MS $>03 S$ BD2 SOLL & 54 \\
\hline $\cos 7$ & osceac & $09 \angle S A L$ & $\Xi N$ JN MN $>0 \Xi S B D Z$ SOLI & 8 \\
\hline Ozop & $0662 \sigma$ & OpLSLLP & BN MS MS $>03 S$ JOZ SOL & 8 \\
\hline oret & ocgzac & $O L L C D \angle Y$ & 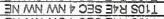 & CL \\
\hline OZTh & $0 \angle 6 Z 9 S$ & 02591.47 & 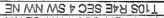 & 58 \\
\hline & & & MN $3 S$ MN । DIS MZY SFII & 8 \\
\hline & & & MN $\Xi S M N \perp$ SS MZZ S\$LI & 8 \\
\hline OSSt & $09 \operatorname{ccos}$ & $O C L S \mid \angle D$ & IN $3 N$ MS $\gg$ O JS $\exists \ngtr P$ SOL 1 & 8 \\
\hline OZSY & Co. & $08991 . \angle 0$ & $\exists N$ IN MS $>$ OES $3 \not Z C$ SOL & 8 \\
\hline onet & ocazas & OLLS.LD & BN MN MN $>0 \exists S$ J6Y SOL. & 8 \\
\hline $\cos 7$ & 066285 & $000 \angle L \angle P$ & 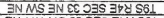 & 8 \\
\hline Co9t & 026289 & $012 \angle b \angle 9$ & JN MN MS CE J3S $\exists \square Y$ SG 1 & S'L \\
\hline 0097 & OSOEDS & $O L L S B \angle B$ & 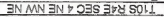 & g. 9 \\
\hline $\cos 5$ & 025289 & OStLLLG & IN MN MS EE OBS $3 \nabla Y$ S61 & 8 \\
\hline 009 & 066295 & 00024268 & 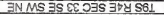 & 8 \\
\hline 0098 & 066289 & $000 \angle . \angle 9$ & IN MMS JN EC OBS $\exists \square Y$ S SGL & 8 \\
\hline 0097 & 068296 & $08<\angle 4 \angle 6$ & ZNMS $\equiv N$ CE 3 S 378 S61 & 8. \\
\hline 099 & 066289 & OOELLLY & JN MS JS EE OSS JVU SEL & s \\
\hline $\cos \%$ & 066239 & $0 Z Z \angle L \angle \square$ & JN MS $\Xi S$ CE OJS JPY्व SSL & 8 \\
\hline $00 S 7$ & 086289 & $00 \% \angle L \angle Y$ & 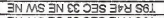 & 8 \\
\hline $0 / 5 \%$ & OCOCE9 & 0004167 & 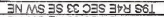 & cis \\
\hline $\cos p$ & DGGCSG & $\cos L 12$ & 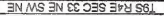 & 8 \\
\hline $\cos t$ & 000089 & $0,0991.6$ & 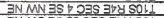 & 2 \\
\hline $0 \angle 99$ & 090889 & $050 \angle 2 . \angle P$ & SNMS $3 S$ c S3S 378561 & 5 \\
\hline 009 & 076289 & 04.4 .49 & 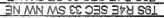 & $\frac{1}{L}$ \\
\hline $\cos \%$ & 080089 & cossitip & 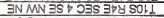 & $L$ \\
\hline 0090 & CQZFOS & 00991.49 & IS JN MN EEO & 8 \\
\hline 0009 & $0569 / 9$ & OCQ5OLL & SN $\Xi S$ MN Z OSS JEY SIL1 & SLL \\
\hline 0097 & 02059 & $09 \angle 9142$ & $\exists N$ IN $M N+O \exists S$ J $\exists$ d SOLI & 9 \\
\hline OZH & OSSZSE & $0 \operatorname{css} \angle 9$ & IN MN MS $\square$ OJS $\exists \forall Y$ SOLI & s'g \\
\hline Colv & $0, \angle 289$ & $0 \angle 091 \angle 7$ & 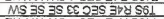 & $\frac{1}{4}$ \\
\hline costo & 000889 & $0005 i L 9$ & $\exists N M N M S$ Y OJS $\exists Y Y$ SOLI & S'L \\
\hline ont & $\cos 289$ & ODDG1L & 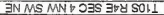 & Ob \\
\hline 0097 & coess & $05 \angle S D \angle 9$ & JN IN MN $\square$ OAS 304 SOLI & $L$ \\
\hline cosp & $0 \angle O$ & OZBSLLP & BN MN IN $~ O \exists S$ JVY SOLL & $-\frac{2}{8}$ \\
\hline 0094 & $0 \angle 0489$ & OESGSLL & SN MWN $Z N \rightarrow O$ & 6 \\
\hline 009 & 090505 & Orosiz & SN MN SN У OAS $37 Y$ SOL & 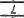 \\
\hline $009 \%$ & Oद्वानुS & $O L \angle S W L D$ & IN MN $3 N \rightarrow$ SES $3 \forall 4$ SOl 1 & 8 \\
\hline 0096 & 00258 & OSLSLLZ & 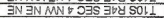 & 8 \\
\hline 009 & 001299 & cosGi:L & 7035304 & 9 \\
\hline OZS & OELES: & $08996 \angle$ & 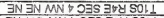 & $L$ \\
\hline Onth & COSZBS & OriGIL & BN MS NWN $D$ OES $\exists G Y$ SOL1 & $B$ \\
\hline $0 \rightarrow 95$ & 097289 & $0.8 t / h$ & उक्ष sol 1 & $\frac{L}{L}$ \\
\hline $0 \rightarrow 96$ & 092285 & $0,00961<9$ & OSS $\exists$ ryd S6I & 8 \\
\hline DOS & 0e0ess & $0 \cos 515$ & 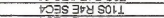 & 8 \\
\hline 002 & $0 \angle 29289$ & 0085127 & (1) & $\frac{5}{L}$ \\
\hline ogen & & $09 \angle 2+\angle P$ & 45011 & L \\
\hline $009 \%$ & & OSLLLV & 1501.1 & 8 \\
\hline $0 \rightarrow 6$ & $0968 \angle 9$ & $009 Z \backslash \angle D$ & 81035 STY SOL. & Ob \\
\hline of & 096819 & $0092 / \angle 7$ & 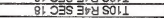 & 8 \\
\hline & & & MWESMH & S. \\
\hline $008 \%$ & OSER8 & OSLGLLD & $B N B N M$ & $\frac{L}{L}$ \\
\hline ozBz & 001509 & $0 \cos \cos \angle 5$ & MSMS $3 S Z Z O J S \exists \forall Y S L$ & $\frac{2}{8}$ \\
\hline 9996 & $\cos$ & $09 B 0 \& \angle D$ & MSMNGS VIJ & S \\
\hline 2902 & & 0906815 & SS MS MS EZ JIS JTY SLI & L \\
\hline 2992 & & $0806 E \angle 9$ & 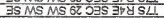 & $\frac{2}{9}$ \\
\hline $081 \varepsilon$ & & $088 Z E \angle G$ & 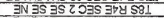 & 9 \\
\hline Oदाह & & $\operatorname{cosec} L$ b & 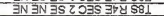 & 9 \\
\hline 0095 & $0 \cos 859$ & $00991 . \angle 7$ & BN $3 N$ IN Y OSS 3 G SOL 1 & s \\
\hline 0097 & & OSLGLLF & $\exists N$ JN MN $>$ OES $3 \triangleright Y$ S SOLL & L \\
\hline $0 \leqslant / 2$ & & & G2S SL JVy & $\frac{2}{L}$ \\
\hline $0 G \angle Z$ & Sol6g & $0 S E L L D$ & $\exists S M N$ SZS S $\angle I F Y$ & \\
\hline OGLL & & $0089 E \angle A$ & SSL उमd & c \\
\hline$O G L Z$ & $0 \angle 2895$ & ORELELF & IS MN GZS SLL $36 Y$ & 9 \\
\hline$O S L Z$ & $0 \angle 8 Y \angle 9$ & $0, \angle \angle B L 9$ & L233s jeas91 & L \\
\hline
\end{tabular}




\begin{tabular}{|c|c|c|c|c|}
\hline OFE & 088289 & OESGLLY & 3N MNN MN D 035 SBD SOLL & $\Rightarrow$ \\
\hline Oन्ह & 088285 & $0 \angle L C L \angle Y$ & 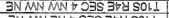 & s \\
\hline OzC & OELICS & C8991/4 & 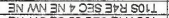 & c \\
\hline $00 g^{2}$ & 060209 & $00 \% \angle 6 \angle 6$ & 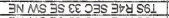 & 7 \\
\hline 0997 & 086289 & 0724218 & SNASS SS EE OAS JIYUS61 & SD \\
\hline 089 & OZLEBS & $00191 / 4$ & IS SN MN EESBS J6Y SB1 & ç \\
\hline$O L S T$ & OSOCBS. & $090 \angle L \angle y$ & JNMS $\exists S$ EE OJS $\exists+4$ S 61 & c) \\
\hline 0857 & OZLEBS & $00 \angle 91 \angle 7$ & 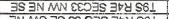 & s \\
\hline OLSP & $090 \mathrm{CBS}$ & 0604.26 & JNMS $\exists S$ \&E OJS $\exists$ H S6 1 & 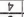 \\
\hline $0 \angle S O$ & 080589 & $0,0,4217$ & $\exists S M N$ JN EE $O 7 S$ JดV S61 & G \\
\hline $\cos p$ & O6SZSS & $000 \angle L \angle>$ & 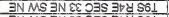 & 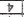 \\
\hline Cogt & 028295 & $O B Z L L L$ & IN M S JN CE $J \exists S \quad \exists \neg \bar{Z}$ S6L & $i$ \\
\hline 029 & 026299 & $0 \angle 19 L \angle D$ & SNMS MS $\gg 03 S$ J7Z SOL 1 & 9 \\
\hline $08 E$ & OLOZOS & $00 \angle \mathrm{SIL} \angle>$ & IN MN MS $\rightarrow 0$ SS JBVU SOLI & SD \\
\hline $08 c t$ & $0 \angle 8289$ & $00 \angle 9 L \angle 7$ & 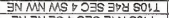 & c'b \\
\hline ots & DSTESS & $O P L S L \angle P$ & 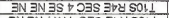 & s's \\
\hline 0097 & Ozecas & $09 \angle G L \angle D$ & BN SN MNV OES $\exists B Y$ S SOLL & s \\
\hline 0097 & OLOESG & OLLSIL & BNMN $\exists N D O Z S$ OPO SOL1 & $9 \overline{1}$ \\
\hline OBE & $0 \angle 8 Z S S$ & $0 O \angle S D \angle D$ & BN MN MS $>0 \exists S$ JPV SOLI & s't \\
\hline 0982 & OZEZOQ & $000 \angle E L F$ & MS MS MN BZ OBS $\exists \square Z$ SU & $s+$ \\
\hline 0882 & $080 Z 69$ & $0 \leq 0 \angle C L>$ & MS MS MN $8 Z$ O $3 S$ JणY SLI & $s \rightarrow$ \\
\hline 0882 & 092289 & $080 \angle E L D$ & MS MS MN BZ OES JWU SLI & 7 \\
\hline cosez & $0 \operatorname{0Ec89}$ & ODOLELT & 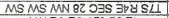 & $g^{\prime}$ \\
\hline gSOE & $00 \mathrm{COLS}$ & OSTOELI & 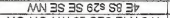 & s \\
\hline 0182 & OOEZRG & $09998 \angle 7$ & MS MS MN & s \\
\hline 0082 & DEvos & OZBFEL & MS MS MS SOAS $\exists$ YY SOLI & 7 \\
\hline SS9E & $0029 / 5$ & 0960ELP & MN $3595625593 y$ & 9 \\
\hline gSGE & DOE9L9 & OSFOCLL & MN $\exists S \exists S 62^{\circ} S 8 \exists y$ & $b$ \\
\hline 0002 & 00 ros & OzSTELA & MSMS MS SOAS JGY SOLI & 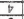 \\
\hline 9992 & 002919 & Co $\% O E L Y$ & MN $3 S 3 S 6 Z^{\circ} S 8^{\prime} \exists$ & $\frac{9}{9}$ \\
\hline $00 B Z$ & 096189 & SZOLELF & MS MS MN 82 J $\exists S$ उYZ & sp \\
\hline OLgr & OStegs & ot $\angle S 1 / 8$ & $\exists N$ JN $3 S \nabla 23 S$ S 30 \& SOLL & 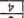 \\
\hline 0096 & 0,0695 & OLLSLLS & 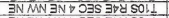 & $\mathrm{s}$ \\
\hline 0090 & 0obicgs & OLLGLP & 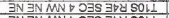 & gg \\
\hline 0.97 & 0 orress & $O B \angle S B \angle B$ & JN $3 N$ MN $D 09 S$ Jb8 SOLL & sp \\
\hline 0,95 & Orfese & $O P L C L \angle D$ & BN $3 N$ IS $+03 S$ BVD SOL & $\underline{c}$ \\
\hline oter & 028285 & $0 \angle L L \angle P$ & 3N MN MN $P O B S$ JPY SOL1 & $\mathrm{s}$ \\
\hline $009 \%$ & 08209 & $08 \angle G, \angle P$ & $3 N 3 N$ MN $603 S 368$ SOLI & G \\
\hline $08 \mathrm{CP}$ & $0 \angle 8 Z B S$ & $00 \angle C L \angle P$ & $\exists N$ MN $M S \rightarrow O \exists S$ BP & s't \\
\hline OBED & $O L 8289$ & $00 \angle S L \angle P$ & IN MNMS $\rightarrow 2 \exists S$ J十Z SOL & c \\
\hline $009 p$ & 081685 & $O \angle \angle S \angle D P$ & $3 N$ IN MN $D O \exists S$ J68 SOLL & 97 \\
\hline 0.95 & CSTEBG & OrLCLS & SN $\exists N$ IS $\square O J S$ JVZ SOLI & S' \\
\hline 0095 & Dobics & $0 \angle L C L L$ & $\exists N$ BN MN D O & 9 \\
\hline 008 & & $06 \angle S L L P$ & BN $3 N$ MN $>0$ OS $30 Y$ SOLL 1 & 5 \\
\hline $09 \angle 2$ & 018889 & $09 \angle S E L Y$ & IS MN GES SL JणV & st \\
\hline $009 \%$ & Dazcos & $08 / 9, \angle 5$ & 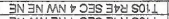 & s \\
\hline $\cos \%$ & OLOEBS & OLLSILT & 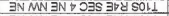 & 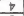 \\
\hline 0097 & 090289 & $01091 \angle 6$ & IN MN $\exists N D$ OJS $\exists T C$ SOLL & 5 \\
\hline 0096 & 0,0009 & $01695 \angle 7$ & 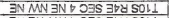 & 9 \\
\hline 0099 & 002899 & $09 / 9127$ & BN $3 N$ MN $\nabla 03 S$ J72 SOL1 & S.7 \\
\hline 0090 & 092889 & $06 \angle S L \angle D$ & 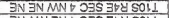 & ç \\
\hline $003 p$ & OLOESG & $\cos 51 / 2$ & 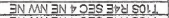 & SD \\
\hline $\cos { }^{\circ}$ & $0 / 0$ ES & OLLSIV & 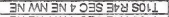 & cib \\
\hline $009^{\circ}$ & Do1sac & $0 \angle L S \angle Q$ & ZN $3 N$ MN P J SS J十O SOL1 & 56 \\
\hline 029 & OELE⿱ES & OEgCLLD & SN MN MS VO OBS $\exists \square Z$ SOL. & $b$ \\
\hline 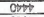 & OOSZES & OPGG.LP & 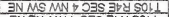 & s \\
\hline 0090 & 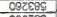 & OSLChLA & IN JN MN 0 OES SBY SOLI & $s p$ \\
\hline onth & 006289 & Clachly & BN MS MNI OAS J十U SOLL & S' \\
\hline $009 p$ & 0,05089 & $\cos 8 \log \angle \theta$ & 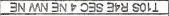 & $\rightarrow$ \\
\hline Ozpt & Olo6s & $06991 / 7$ & 80353 3 & G \\
\hline 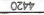 & OlOEgs & 00991.45 & $6035 \exists 7045011$ & 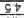 \\
\hline 0095 & CSERES & DOLSLLD & 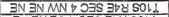 & g's \\
\hline $0, S^{\circ}$ & 097289 & OFLSLLG & IN $3 N \exists S+03 S$ J Y SOLI & $b$ \\
\hline 0099 & CSESOS & $0 S \angle S L \angle P$ & BN $\exists N M N \triangle O Z S$ BDY SOL1 & 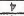 \\
\hline 0190 & 0otsos & $O F \angle G \backslash \angle Q$ & 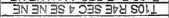 & 7 \\
\hline OESY & & $O E \angle G B L$ & BNMN $3 N \nabla 3$ & G \\
\hline 0096 & 081805 & $0.152 \angle B$ & BN BN MN $\rightarrow$ OSS SBY SOLL & s \\
\hline OZS & OClcos & 0899120 & 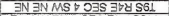 & 5 \\
\hline $00 \theta^{\circ}$ & $090 \mathrm{cos}$ & $0+896.20$ & 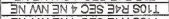 & 7 \\
\hline OZSY & OSIES & $0699 . \angle D$ & 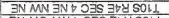 & G. \\
\hline 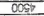 & OBOCBS & $06991 / 4$ & BN MS MN $D O E S$ Jo SOL 1 & 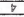 \\
\hline CONt & $O \angle B E O S$ & $0299 / \angle 4$ & JNMNANS $P$ OAS JPY SOL1 & $\$$ \\
\hline 000 & OSOEST & Ong9. 49 & 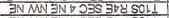 & $t$ \\
\hline 0289 & OEIESG & OQ9961 $\angle 8$ & 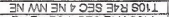 & $\mathrm{s}$ \\
\hline OZS & CSIEBS & $09951 / 2$ & SN $B N B S \square O B S B \square \square C$ & $\mathrm{~s}$ \\
\hline DEST & OEIEBS & $00991 \angle 7$ & $3 N M N B N \rightarrow O B S$ BVY SOLL & 5 \\
\hline 0096 & 090889 & Orogilla & BN MN JN $\triangle O B S$ JVY SOLA & g \\
\hline 000 & costres & $01 C 51 \angle 7$ & DSES $360 \mathrm{~d}$ SOL. 1 & $g b$ \\
\hline 002 & $0 \angle 9 Z 89$ & $0969, \angle 7$ & 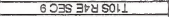 & c \\
\hline 0028 & $0 \angle 2789$ & $09395 \angle 7$ & DOSS J7a soll & c \\
\hline orat & 097289 & $0.8 \% L \angle A$ & Dojs joud SOL1 & $g b$ \\
\hline orai & 005289 & OLSD $L A$ & $82035 \exists 52561$ & $\rightarrow$ \\
\hline 0027 & OLOZOS & OOBSILD & DOSS at2 SOL1 & 5 \\
\hline 0026 & $0 \angle 2289$ & $00095 \angle 6$ & vojs JPa SOLI & G \\
\hline opsis & 097289 & ONTF, L & 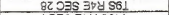 & gis \\
\hline Orst & $09 \div 209$ & $0, E>1 L 7$ & PDES $3+8 \mathrm{SOH}$ & $\underline{G \rightarrow}$ \\
\hline OFS5 & $\cos 209$ & 02016 & 82035378581 & $t$ \\
\hline 0099 & 0,2789 & 0.90215 & 9. OJS $\exists Z 4$ S61 & 7 \\
\hline 0790 & 09828 & 068064 & 55383725011 & s7 \\
\hline $\cos 9$ & DOZTS9 & Ol.902/7 & 91038 जटd 561 & 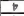 \\
\hline $\cos 5$ & $0288 \angle C$ & C9L2,L7 & 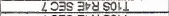 & 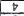 \\
\hline $00 \sin$ & 028815 & colzalt & $\angle O A S$ JFY SOLL & 8 \\
\hline $00 \mathrm{n}$ & $0068 \angle 5$ & OSSELLP & $\angle O B S$ BPO 5011 & 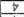 \\
\hline 095 & $0,89 \angle 5$ & $092 Z 1, \angle B$ & $\angle O \equiv S \exists Y{ }^{2}$ SOLL & 17 \\
\hline
\end{tabular}




\begin{tabular}{|c|c|c|c|c|}
\hline $06 / 18 / 95$ & 554 & Uta & stansounana & $\mathrm{m}$ \\
\hline $06 / 21 / 95$ & 1635 & Uta & stansbunang & $r$ \\
\hline $06 / 21 / 95$ & 2012 & Uta & stansounana & $r$ \\
\hline $0,5 / 24 / 95$ & 11143 & Uta & stansbunana & 1 \\
\hline $06 / 24 / 95$ & 1147 & Uta & stansbunana & 1 \\
\hline $06 / 24 / 95$ & 1214 & Uta & stansburiana & 1 \\
\hline $06 / 24 / 85$ & 1104 & Uta & stanebunana & $m$ \\
\hline $06 / 26 / 95$ & 1838 & Uta & stansburiang & $m$ \\
\hline $06 / 26 / 95$ & 1920 & Uta & stansbunana & $m$ \\
\hline $06 \sqrt{26 / 95}$ & 1235 & Una & stansbunana & $r$ \\
\hline $06 / 26 / 95$ & 1823 & Uta & stansbunana & $m$ \\
\hline $05 / 26 / 95$ & 1221 & Uta & stansbunana & i. \\
\hline $06 / 26 / 95$ & 11235 & Uta & stansbunana & r \\
\hline $06 / 26 / 93$ & 1235 & Uta & stansbunana & 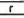 \\
\hline $06 / 27 / 95$ & 1900 & Uta & stans buniana & 1 \\
\hline $05 \sqrt{28 / 95}$ & 1026 & Uta & stansburiana & $r$ \\
\hline $08 / 28 / 95$ & 1654 & Uta & stansbunans & $m$ \\
\hline $05 / 28 / 95$ & 1115 & Uts & stansburiana & 1 \\
\hline $06 / 28 / 95$ & 1058 & Uta & stansbunana & 1 \\
\hline $0,128 / 95$ & 1032 & Uita & stansbunana & 1 \\
\hline $06 / 28 / 95$ & 1032 & Uta & stansburiana & $r$ \\
\hline $05 \sqrt{28 / 95}$ & 1323 & Uta & stansburiana & 1 \\
\hline $06 / 28 / 95$ & 11148 & Uia & stansouniana & $b$ \\
\hline $06 / 29 / 95$ & 948 & Uta & stansouriana & $i$ \\
\hline $06 / 29 / 95$ & 1148 & Uta & stansburiana & $i$ \\
\hline $07 / 01 / 95$ & 1837 & Uta & stansouriana & 1 \\
\hline $07101 / 95$ & 1956 & Uta & stansounana & 1 \\
\hline $07101 / 55$ & 2001 & Uth & Stansbunana & $i$ \\
\hline $07 / 01 / 95$ & 1900 & Ute & stansouriana & 1 \\
\hline $07101 / 95$ & 1900 & Uta & stensburians & 1 \\
\hline 07102155 & 1515 & Uta & stansbunaria & $m$ \\
\hline $07 / 02 / 95$ & 945 & Uta & stansburiana & 1 \\
\hline $07 / 05 / 95$ & 2021 & Uta & stansburiana & $i$ \\
\hline $07 / 05 / 95$ & 2023 & Uta & stansburiana & 1 \\
\hline $07108 / 95$ & 1111 & Uta & stansburiana & 1 \\
\hline $0710 \mathrm{~B} / 95$ & 1111 & Uta & stansbunana & $t$ \\
\hline $07 / 108 / 95$ & 11111 & Uta & stansburiana & $r$ \\
\hline 0711295 & 1318 & Uta & stansbunang & $r$ \\
\hline $07 / 15 / 95$ & 11147 & Uta & stansounana & $r$ \\
\hline $07 / 15 / 95$ & 11157 & Uta & stansbunana & $\mathrm{b}$ \\
\hline $07 / 15 / 95$ & 1155 & Uta & gtansburiang & $b$ \\
\hline $07 / 15 / 95$ & 1157 & Uta & stansourians & 0 \\
\hline $07 / 15 / 95$ & 1245 & Uta & stansbuniane & i \\
\hline $07 / 15 / 95$ & 1200 & Ula & gtansburiana & $b$ \\
\hline $0715 / 95$ & 11124 & Ute & stansouniana & $b$ \\
\hline $07 / 15 / 95$ & 1320 & Uta & stansburians & $r$ \\
\hline $07 / 15 / 95$ & 828 & Ula & stansbuniana & 1 \\
\hline $07 / 15 / 95$ & 1217 & Uta & stansburiana & $r$ \\
\hline $07 / 18 / 95$ & 11130 & Uta & stansburiana & $r$ \\
\hline $07 / 18 / 95$ & 1135 & Ula & stensbunane & $r$ \\
\hline $07 / 18 / 95$ & 1107 & Uta & stansbunana & $r$ \\
\hline $07 / 18 / 95$ & 1110 & Uta & stansbunana & $r$ \\
\hline $07 / 25 / 95$ & 1036 & Ut: & stansounana & m \\
\hline $07 / 29 / 95$ & 644 & Uta & stansouniana & $t$ \\
\hline $07 / 29195$ & 643 & Uta & stansbunana & $i$ \\
\hline $09 / 02 / 35$ & 914 & Uta & stansburiana & $\mathrm{m}$ \\
\hline $09 / 02 / 95$ & 830 & Uta & stansbunana & i \\
\hline $09 / 06 / 95$ & 1612 & Uta & stansbunana & $r$ \\
\hline $09 / 12 / 95$ & 815 & Uia & stansburiana & $r$ \\
\hline $09 / 16 / 95$ & 848 & Uta & stansbunana & $r$ \\
\hline $09 / 19 / 95$ & 809 & Uta & stansbunana & $r$ \\
\hline $09 / 23 / 95$ & 1744 & Uta & gtansbunana & $r$ \\
\hline $05 / 28 / 95$ & 2000 & Uta & stansburiana & $r$ \\
\hline $07 / 03 / 95$ & 1850 & Uta & stansbunana & $r$ \\
\hline $07 / 03 / 95$ & 1848 & Ute & stansbunana & $t$ \\
\hline $07 / 103 / 95$ & 1829 & Via & stansbunang & $i$ \\
\hline Sngkes & & & & \\
\hline $05 / 18 / 95$ & 1442 & Coluber & constrictor & $i$ \\
\hline $05 / 10 / 95$ & 1811 & Coluber & constrictor & $i$ \\
\hline $08 / 12 / 95$ & 1737 & Coluber & constrictor & $i$ \\
\hline $08 / 12 / 95$ & 1737 & Coluber & constrictor & $i$ \\
\hline $0,5 / 20 / 95$ & 1410 & Coluber & constnctor & $r$ \\
\hline $05 \sqrt{20195}$ & 1647 & Coluber & constrictor & b \\
\hline $06 / 20 / 95$ & 1753 & Coluber & constrictor & $\mathrm{b}$ \\
\hline $06 / 26 / 95$ & 914 & Coluber & constrictor & $i$ \\
\hline $07 / 03 / 95$ & 1812 & Coluber & constrictor & $r$ \\
\hline $07108 / 95$ & 1103 & Coluber & constrictor & $i$ \\
\hline $07102 / 55$ & 1528 & Coluder & constrictor & $t$ \\
\hline $07 / 09 / 93$ & 1522 & Coluber & constictor & $i$ \\
\hline $07 / 12 / 35$ & 1136 & Coluber & constrictor & $b$ \\
\hline $07 / 23 / 25$ & 1132 & Coluber & constrictor & $\mathrm{I}$ \\
\hline $09 / 12 / 95$ & 852 & Coluber & constrictor & $i$ \\
\hline $05 / 18 / 95$ & 1644 & Crotalus & vinis & $i$ \\
\hline $05 / 1895$ & 1721 & Crotalus & Mindis & 1 \\
\hline $05 / 20185$ & 1000 & Crotalus & viricis & 1 \\
\hline $05 / 21 / 85$ & 814 & Crotalus & viridis & 1 \\
\hline $05 / 22 / 35$ & 1800 & Crotalus & vindis & $t$ \\
\hline $05 / 22 / 85$ & 11107 & Crotalus & nnindis & $i$ \\
\hline $05 / 28195$ & 1950 & Crotalus & Mnidis & $t$ \\
\hline $05 / 30 / 95$ & 1154 & Crotalus & vilidis & $m$ \\
\hline $05 \sqrt{30195}$ & 1225 & Crotalus & nnidis & $t$ \\
\hline $05 / 30 / 95$ & 1203 & Crotalus & nindis & I \\
\hline $06 / 08 / 95$ & 1030 & Crotalus & viníis & $r$ \\
\hline $06 / 13 / 95$ & 1956 & Crotalus & vindis & $r$ \\
\hline
\end{tabular}




\begin{tabular}{|c|c|c|c|c|c|}
\hline $06 / 14 / 95$ & 1800 & Crotalus & viridis & $r$ & 40 \\
\hline $06 / 14 / 95$ & 1238 & Crotalus & viridis & i & no \\
\hline $06 / 14 / 95$ & 1729 & Crotalus & vindis & r & 39 \\
\hline $06 / 495$ & 1910 & Crotalus & vindis & 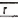 & 43 \\
\hline $06 / 14 / 95$ & 1103 & Crotalut & vinidis & $r$ & 38 \\
\hline $06 / 14 / 95$ & 1810 & Crotalus & vindis & $r$ & 41 \\
\hline $06 / 15 / 95$ & 1111 & Crotalus & vindis & 1 & no \\
\hline $06 / 19 / 95$ & 1145 & Crotalus & vinidis & $r$ & no \\
\hline $06 / 20 / 95$ & 1711 & Crotalus & vindis & 1 & no \\
\hline $05 \sqrt{21 / 95}$ & 1630 & Crotalus & vindis & $r$ & 40 \\
\hline $06 / 24 / 95$ & 2100 & Crotalus & vindis & $i$ & no \\
\hline $08 / 24 / 95$ & 958 & Crotalus & viridis & $m$ & 50 \\
\hline $05 / 27 / 95$ & 940 & Crotalus & जnidis & $T$ & 52 \\
\hline $06 / 27 / 95$ & 1822 & Crotalus & vinidis & i & 53 \\
\hline $06 / 28 / 95$ & 1255 & Crotalus & vinidis & $r$ & 55 \\
\hline $06 / 29 / 95$ & 1030 & Crotalus & vindis & $i$ & no \\
\hline $07 / 01 / 95$ & 1950 & Crotalus & vinidis & 8 & 40 \\
\hline $07 / 02 / 95$ & 934 & Crotalus & vinidis & 1 & 53 \\
\hline $07 / 03 / 95$ & 1345 & Crotalus & vinidis & $i$ & no \\
\hline $07 / 05 / 95$ & 2211 & Crotalus & vinidis & 1 & 40 \\
\hline $07 / 05 / 95$ & 2039 & Crotalus & vindis & $\frac{1}{r}$ & 41 \\
\hline $07 / 08 / 95$ & 1112 & Crotalus & vindis & $r$ & $\frac{41}{40}$ \\
\hline $07 / 12 / 95$ & 853 & Crotalus & vindis & $i$ & no \\
\hline $07 / 15 / 95$ & 1205 & Crotalus & vinidis & r & 39 \\
\hline $07 / 15 / 55$ & 1320 & Crotalus & vininis & r & 86 \\
\hline $07 / 1805$ & 1000 & Crotalus & Viridis & $b$ & 89 \\
\hline $09 / 09 / 95$ & 1200 & Crotalus & vinidis & $r$ & no \\
\hline $05 / 21 / 95$ & 820 & Hypseglena & torquata & r & 6 \\
\hline $05 / 27 / 95$ & 1121 & Hyoseglena & torquata & $r$ & 8 \\
\hline $06 / 01 / 95$ & 2046 & Hypsegiena & torquata & b & no \\
\hline $06101 / 95$ & 1858 & Hypseglena & torquata & 1 & 17 \\
\hline $07103 / 35$ & 1147 & Hypseglena & torquata & 1 & no \\
\hline $07 / 103 / 95$ & 1147 & Hypseglens & torguata & $i$ & no \\
\hline $08 / 15 / 95$ & 830 & Hypsipiena & torquata & $\mathrm{m}$ & 45 \\
\hline $06 / 27 / 95$ & 1250 & Hypsiglana & torquate & $b$ & no \\
\hline $07 / 03 / 55$ & 19399 & Hypsiglena & torquata & $i$ & no \\
\hline $09 / 23 / 95$ & 1842 & Hypsigiena & torquata & $r$ & no \\
\hline $08 / 26 / 95$ & 900 & Hypsiglena & torquata & r & no \\
\hline $05 / 18 / 95$ & 1558 & Masticophis & taeniatus & $i$ & no \\
\hline $05 / 19 / 95$ & 1030 & Masticophis & taeniatus & $i$ & no \\
\hline $05 / 21 / 95$ & 1923 & Masticophis & taeniatus & 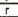 & 3 \\
\hline $05 / 27 / 95$ & 11209 & Masticophis & taoniatus & $\mathrm{m}$ & 9 \\
\hline $05 / 27 / 85$ & 19242 & Masticophis & taeniatus & 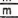 & 12 \\
\hline $06 / 01 / 95$ & 17712 & Masticophis & taeniatus & $i$ & $\frac{12}{n o}$ \\
\hline $06 / 05 / 95$ & 1847 & Masticophis & taeniatus & $r$ & 23 \\
\hline $06 / 06 / 95$ & 1356 & Masticophis & taeniatus & $i$ & no \\
\hline $06 / 10 / 95$ & 2133 & Mastisophis & taeniatus & $r$ & 28 \\
\hline $06 / 13 / 95$ & 938 & Masticophis & taeniatus & $r$ & 3 \\
\hline 0611495 & 1728 & Masticophis & taeniatus & 1 & 3 \\
\hline $05 / 18 / 95$ & 1004 & Masticophis & taeniatus & $r$ & no \\
\hline $06 / 24 / 95$ & 1230 & Masticoohis & taeniatus & p & no \\
\hline $06 / 26 / 95$ & 935 & Masticophis & taeniatus & 1 & no \\
\hline $06 / 26 / 95$ & 836 & Masticophis & taeniatus & $i$ & no \\
\hline $06 / 26 / 95$ & 1242 & Mastioophis & taeniatus & 1 & 51 \\
\hline $05 / 27 / 95$ & 1852 & Masticophis & taeniatus & $r$ & 54 \\
\hline $05 / 28 / 95$ & 1226 & Masticophis & taeniatus & 1 & 54 \\
\hline $07 / 01 / 95$ & 1839 & Masticophis & taeniatus & 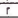 & 60 \\
\hline $07 / 01 / 95$ & 1912 & Masticophis & taeniztus & $\frac{1}{1}$ & 63 \\
\hline $07 / 02 / 95$ & 1303 & Masticophes & taeniatus & b & no \\
\hline $07 / 102 / 55$ & 1025 & Masticophis & taeniatus & $\mathrm{b}$ & no \\
\hline $07 / 03 / 95$ & 1147 & Masticophis & taeniatus & $i$ & no \\
\hline $07 / 03 / 95$ & 1750 & Masticophis & taeniatus & i & 67 \\
\hline $07 / 03 / 95$ & 1735 & Masticoghis & taeniatus & $r$ & 60 \\
\hline $07 / 103 / 85$ & 1449 & Masticophis & taeniatus & $i$ & no \\
\hline $07 / 103 / 95$ & 1046 & Masticophis & taeniatus & r & 72 \\
\hline $07 / 08 / 95$ & 1302 & Masticophis & taeniatus & $\frac{1}{1}$ & 28 \\
\hline $07 / 08 / 95$ & 1026 & Masticophis & taeniatus & $r$ & no \\
\hline $07 / 12 / 95$ & 1156 & Masticophis & taeniatus & $\frac{1}{b}$ & no \\
\hline $07 / 15 / 95$ & 830 & Mesticophis & taeniatus & 1 & 28 \\
\hline $07 / 15 / 95$ & 1320 & Masticophis & taeniatus & i & 85 \\
\hline $07 / 18 / 95$ & 1120 & Mesticoohis & taeniatus & 1 & 91 \\
\hline $07 / 18 / 95$ & 1145 & Masticophis & taeniatus & I & 92 \\
\hline $07 / 21 / 95$ & 1712 & Masticophis & taeniatus & $r$ & $\frac{45}{25}$ \\
\hline $07 / 23 / 95$ & 1051 & Masticophis & taeniatus & $i$ & no \\
\hline $07 / 23 / 95$ & 1041 & Masticophis & taeniati & $i$ & no \\
\hline & 820 & Masticophis & taeniatus & 1 & 25 \\
\hline $09 / 15 / 95$ & 11605 & Mesticophis & taeniatus & $\frac{1}{r}$ & 101 \\
\hline $09 / 12 / 35$ & 815 & Masticophis & taeniatus & 1 & 104 \\
\hline $09 / 16 / 95$ & 800 & Mesticophis & taeniatus & 1 & 63 \\
\hline $09 / 19 / 95$ & 845 & Masticophis & taeniatus & $m$ & no \\
\hline $09 / 19 / 95$ & 809 & Masticophis & taeniatus & $\frac{1}{1}$ & no \\
\hline $09 / 23 / 95$ & 1815 & Masticophis & taeniatus & r & 55 \\
\hline $09 / 23 / 95$ & 1755 & Masticophis & taeniatus & $r$ & no \\
\hline $09 / 23 / 95$ & 1842 & Masticophis & taenigtus & $r$ & no \\
\hline $05 / 18 / 95$ & 1603 & Pituophis & catenifer & $i$ & no \\
\hline $05 / 18 / 95$ & 1522 & Piuophis & cateniter & $i$ & no \\
\hline $05 / 18 / 95$ & 1607 & Pituophis & catenifer & $i$ & no \\
\hline $05 / 18 / 95$ & 1609 & Pituophis & catenifer & i & no \\
\hline $05 / 18 / 95$ & 1524 & Pituophis & catenifer & $i$ & no \\
\hline $05 / 18 / 95$ & 1535 & Pituophis & catenifer & $i$ & no \\
\hline $05 / 18 / 95$ & 1600 & Pituophis & catenifer & $i$ & no \\
\hline $05 / 18 / 95$ & 1605 & Pituophis & catenifer & $i$ & no \\
\hline $05 / 18 / 95$ & 1645 & Pituophis & catenifer & 1 & no \\
\hline
\end{tabular}




\begin{tabular}{|c|c|c|c|c|}
\hline $05 / 49 / 95$ & 11158 & Pituophis & cateníor & $m$ \\
\hline $05 / 20 / 95$ & 1252 & Pituophis & catenifer & $i$ \\
\hline $05 / 25 / 95$ & 1208 & Pituophis & catenfer & $\frac{1}{1}$ \\
\hline $05 / 25 / 95$ & 11130 & Pituophis & catenifer & $i$ \\
\hline $05 / 25 / 95$ & 1145 & Pituophis & catenifer & $i$ \\
\hline $05 / 27 / 95$ & 950 & Pituophis & catenifor & $i$ \\
\hline $0,5 / 27 / 95$ & 1238 & Piluophis & catenifer & $m$ \\
\hline $05 / 27 / 95$ & 950 & Pituophis & catenifor & $i$ \\
\hline $08 / 27 / 95$ & 1221 & Pituophis & catenifer & $\mathrm{m}$ \\
\hline $06 / 01 / 95$ & 2051 & Pituoghis & cateníder & b \\
\hline $06101 / 95$ & 11905 & Pituophis & catenifer & i \\
\hline $06 / 01 / 95$ & 2053 & Pituophis & cateniler & $b$ \\
\hline $06 / 04 / 95$ & 1808 & Pítuophis & catenifer & 1 \\
\hline $06 / 01 / 95$ & 2010 & Pituophis & catenifer & $b$ \\
\hline $06101 / 95$ & 12101 & Pituophis & catenter & $b$ \\
\hline $0501 / 5$ & 11824 & Pituophis & catenifer & 1 \\
\hline $06 / 01 / 95$ & 2023 & Pituophis & catenifer & $\frac{1}{b}$ \\
\hline $06 / 04 / 95$ & 2010 & Pituophis & cateniler & $b$ \\
\hline $08 / 05 / 95$ & 1620 & Pituophis & catenifer & $i$ \\
\hline $06 / 06 / 95$ & sos & Pituoonis & catenifer & $i$ \\
\hline $08 / 06 / 95$ & 1743 & Prtugohis & catenifer & $i$ \\
\hline $06 / 06 / 95$ & 1743 & Pituophis & catenifer & $i$ \\
\hline $00 / 10 / 95$ & 1820 & Pituophis & catenifer & $i$ \\
\hline $06 / 10 / 95$ & 2117 & Pituoshis & catenifer & 1 \\
\hline $08 / 10 / 95$ & 1940 & Pituophis & cateniler & i \\
\hline $08 / 10 / 95$ & 1910 & Pituophis & catennier & $i$ \\
\hline $06 / 10 / 95$ & 1832 & Pítuophis & catenifer & $i$ \\
\hline $08 / 10 / 95$ & 1943 & Pituophis & cateniler & $i$ \\
\hline $06 / 10 / 95$ & 1850 & Pituophis & catenifer & $i$ \\
\hline $06 / 11 / 95$ & 1037 & Pituophis & catenifer & $\frac{1}{b}$ \\
\hline $06 / 11 / 95$ & 1130 & Pituophis & catenifer & $m$ \\
\hline$0 5 \longdiv { 1 4 / 9 5 }$ & 1835 & Piltuophis & catenfifer & $r$ \\
\hline $08 / 14 / 95$ & 1038 & Pituophis & catenifer & $\frac{1}{1}$ \\
\hline $05 / 15 / 95$ & 853 & Pituophis & cateniter & $\frac{1}{b}$ \\
\hline $06 / 15 / 95$ & 817 & Pituophis & catenifer & $\mathrm{m}$ \\
\hline $08 / 18 / 95$ & 1915 & Pituophis & catenifer & $i$ \\
\hline $06 / 19 / 55$ & 1408 & Pituophis & cateníler & $i$ \\
\hline $08 / 20 / 95$ & 1936 & Phuophis & catenifer & $i$ \\
\hline $05 / 24 / 95$ & 2045 & Pituophis & catenfer & 1 \\
\hline $08 / 24 / 95$ & 2030 & Piluophis & catenifer & $i$ \\
\hline $08 / 26 / 95$ & 828 & Pituoohis & catenifer & $i$ \\
\hline $06 / 28 / 95$ & 1827 & Privophis & catenifer & $b$ \\
\hline $06 / 29 / 95$ & 808 & Pituophis & catenife: & 1 \\
\hline $07 / 01 / 95$ & 1648 & Pituophis & catenifer & $i$ \\
\hline $07 / 01 / 93$ & 1848 & Pituoghis & catenifer & $i$ \\
\hline $07 / 01 / 95$ & 1903 & Pituophis & catenifer & $i$ \\
\hline $07 / 01 / 95$ & 1637 & Pituophis & catenifer & 1 \\
\hline $07 / 02 / 95$ & 1022 & Pituophis & catenifer & 6 \\
\hline $07102 / 95$ & 1050 & Pituophis & catenifer & 든 \\
\hline $07 / 03 / 55$ & 1739 & Pituophis & catenifer & 8 \\
\hline $07 / 03 / 55$ & 1524 & Pituophis & catenifier & $i$ \\
\hline $07 / 05 / 95$ & 2030 & P'tuophis & catenfier & t \\
\hline $07 / 06 / 95$ & 907 & Pituophia & catenifer & $i$ \\
\hline $07108 / 95$ & 1241 & Pituoghis & catenifer & $\mathrm{m}$ \\
\hline $07 / 08 / 95$ & 917 & Pituoghis & cateniler & $i$ \\
\hline $07 / 12 / 95$ & 11144 & Pituoohis & catenifer & $\frac{1}{b}$ \\
\hline $07 / 15 / 35$ & 910 & Pituophis & catenffer & $\mathrm{m}$ \\
\hline $07 / 15 / 95$ & 1010 & Pituophis & cateniler & $b$ \\
\hline $07 / 15 / 95$ & 1035 & Pituophis & catenifer & b \\
\hline $07 / 18 / 85$ & 800 & Pttuophis & catenffer & $i$ \\
\hline $07 / 18 / 85$ & 745 & Pituophis & catenifer & i \\
\hline $07 / 48 / 95$ & 1015 & Pituophis & catenifier & $b$ \\
\hline $07 / 18195$ & 924 & Pituoghis & catenfief & $m$ \\
\hline $07 / 21 / 95$ & 1951 & Pituophis & catenifer & $m$ \\
\hline $07 / 23 / 95$ & 1954 & Pituophis & catenifer & 1 \\
\hline $07 / 23 / 95$ & 2005 & Piluophis & catenifer & $i$ \\
\hline $07 / 25 / 95$ & 1008 & Pituophis & catenifer & $\frac{1}{b}$ \\
\hline $07 / 29 / 85$ & 810 & Piluophia & catenifor & $m$ \\
\hline $08 / 29 / 95$ & 714 & Pituoghis & catenifer & $i$ \\
\hline $08 / 29 / 95$ & 1000 & Pituophis & catenifier & $i$ \\
\hline $09 / 102 / 95$ & 840 & Pituoohis & catenfer & $t$ \\
\hline $09 / 09 / 95$ & 853 & Pituophis & catenifer & i \\
\hline $09 / 12 / 95$ & 849 & Piltuophis & catenifer & $m$ \\
\hline $08 / 16 / 95$ & 1003 & Pituophis & catenifer & 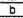 \\
\hline $00 / 23 / 95$ & 1918 & Pituophis & catenifer & $m$ \\
\hline $09 / 23 / 95$ & 19749 & Pituophis & catenifer & $t$ \\
\hline $05 / 21 / 95$ & 19847 & Pituophis & catenifer & $\frac{1}{r}$ \\
\hline $06 / 18 / 95$ & 11851 & Rhinocheilus & leconter & 1 \\
\hline $07 / 03 / 55$ & 1311 & Sonora & semiannulata & $i$ \\
\hline $07 / 03 / 95$ & 19147 & Sonora & \begin{tabular}{|l} 
semiannulata \\
\end{tabular} & $\mathrm{i}$ \\
\hline $07103 / 85$ & 1300 & Sonore & semiannulata & $i$ \\
\hline $07 / 03 / 55$ & 19147 & Sonora & semiannulata & $i$ \\
\hline $07 / 103 / 95$ & 19147 & Sonora & semiannulata & $i$ \\
\hline $07 / 03 / 55$ & 11147 & Sonora & semiannulata & $\frac{1}{1}$ \\
\hline $07 / 03 / 95$ & 11147 & Sonora & semiannulata & $i$ \\
\hline $07103 / 95$ & 1252 & Sonore & semiannulata & $i$ \\
\hline $07 / 03 / 55$ & 19147 & Sonora & semiannulata & $i$ \\
\hline $07 / 03 / 95$ & 1147 & Sonora & semiannulata & $\mathrm{i}$ \\
\hline $07 / 03 / 95$ & 1218 & Sonora & semiannulata & $i$ \\
\hline $07 / 03 / 95$ & 11147 & Sonora & semiannulata & $i$ \\
\hline $07 / 03 / 95$ & 11147 & Sonora & semiannulata & $i$ \\
\hline $07 / 03 / 95$ & 11147 & Sonora & semiennulata & $i$ \\
\hline $07 / 03 / 95$ & 1147 & Sonora & semisnnulata & $\mathrm{i}$ \\
\hline $07 / 03 \sqrt{55}$ & 11147 & Sonora & semiannulata & $i$ \\
\hline
\end{tabular}




\begin{tabular}{|c|c|c|c|c|c|c|c|c|c|c|c|c|c|c|}
\hline $05 / 17 / 95$ & 11423 & Thamnophis & elegans & $b$ & no & & 25 & clear & breezy & 50 & T1OS RAE SEC 18 & 4712810 & 578090 & 4200 \\
\hline $05 \sqrt{30 / 35}$ & 1048 & Thaminophis & elegans & $\frac{1}{b}$ & 14 & $16 \mathrm{C}$ & 25 & clear & breezy & 40 & T9S R4E SEC 33 SE SE & 4716110 & 582680 & 4100 \\
\hline $08 / 01 / 95$ & 2036 & Thamnophis & elegans & $\mathrm{b}$ & 22 & $18: 0$ & 20 & cloudy & breezy & 60 & C. 33 SE SE SW & 4716110 & 582580 & 4100 \\
\hline $06 / 01 / 95$ & 2015 & Thamnophis & elegans & $b$ & no & $15: 6$ & 20 & cloudy & none & 75 & C 33 SE SE SW & 4716040 & 582875 & 4100 \\
\hline $06101 / 95$ & 2028 & Thamnophis & elegans & $b$ & 20 & $16: C$ & $\frac{20}{20}$ & cloudy & broezy & 45 & C 335 SES & 4716110 & 582680 & 4400 \\
\hline $08 / 01 / 95$ & 2031 & Thamnophis & ejegans & $b$ & 21 & $16: H$ & 20 & cloudy & breezy & 45 & T9S R4E & 4716110 & 582680 & 4100 \\
\hline $06 / 08 / 95$ & 1209 & Thamnophis & eiegans & $\bar{b}$ & no & & 17 & cloudy & breezy & 40 & TSS RAE & 4716080 & 582710 & 4160 \\
\hline $06 / 08 / 95$ & 1200 & Thamnophis & elegans & $b$ & no & & 19 & cloudy & Dreezy & 20 & TOS R4E & 4716110 & 582680 & 4100 \\
\hline $06 / 11195$ & 1033 & Thamnophis & elegans & $b$ & 29 & $15: G$ & 26 & clear & none & 45 & TSSR4ES & 4716040 & 582675 & 4100 \\
\hline $06 / 11 / 95$ & 1051 & Thamnophis & elegans & $b$ & 30 & & 23 & clear & none & 45 & TOS R4E SEC 33 SE SE SW & 4716090 & 582710 & 4160 \\
\hline $06 / 13 / 95$ & 1048 & Thamnophis & eleganns & $b$ & 35 & $17: C$ & -25 & clear & breezy & 45 & C 335 W SE SW & 4716120 & 582620 & 4100 \\
\hline $08 / 13 / 95$ & 11048 & Thamnophis & elegans & $\mathrm{b}$ & no & $17: C$ & 25 & clear & breezy & 25 & TSS RAE SEC 33 SW SE SW & 4716120 & 582620 & 4100 \\
\hline $06 / 13 / 95$ & 1004 & Thamnophis & elegans & $b$ & 29 & $16 \cdot \mathrm{B}$ & 26 & clear & breezy & 45 & TOS RAE SEC 33 SE SE SW & $\frac{4110120}{4716110}$ & 582680 & 4100 \\
\hline $06 / 13 / 35$ & 1039 & Thamnophis & elegans & $b$ & 34 & $16: G$ & 26 & clear & broezy & 45 & TOSRLE & 4716110 & 582680 & 4400 \\
\hline $06 / 13 / 95$ & 1033 & Thamnophis & elegans & $\mathrm{b}$ & 33 & $15: 8$ & 26 & clear & breezy & 45 & TOS RAE & 4715040 & 582675 & 4100 \\
\hline $06 / 15 / 95$ & 920 & Thamnophis & elegans & $b$ & 46 & $13 \%$ & 17 & cloudy & breezy & 51 & TOSRAE & 4718090 & 582710 & 4160 \\
\hline $06 / 15 / 95$ & 920 & Thamnophis & elegans & $b$ & 47 & $13: 8$ & 17 & cloudy & breezy & 45 & EC 33 SE SE SW & 4710090 & 582710 & 4160 \\
\hline $06 / 18 / 95$ & 1030 & Thamnoohis & elegans & $b$ & no & $15: \mathrm{A}$ & 17 & cloudy & broezy & 24 & T9S R4E & 4716040 & 582675 & 4100 \\
\hline $06 / 18 / 55$ & 1037 & Thamnophis & elegans & $b$ & 29 & $16: D$ & 26 & clear & breezy & 45 & EC 33 SESESW & 4716110 & 582680 & 4100 \\
\hline $06 / 20 / 95$ & 1024 & Thamnophis & elogans & 1 & no & & $\frac{16}{16}$ & $\pi / c$ & breezy & 25 & EC 16 SW SW SE & & & \\
\hline $06 / 20 / 95$ & 1500 & Themnophis & elegans & $b$ & no & & 16 & $p / c$ & breezy & 45 & EC 1 NW SENW & & & \\
\hline$0 6 \longdiv { 2 4 / 5 5 }$ & 1047 & Thamnophis & elegans & $b$ & 49 & $15: F$ & 28 & clear & breezy & 35 & C 33 SE SE SW & 4716040 & 582675 & 4400 \\
\hline $06 / 24 / 95$ & 1030 & Thamnophis & elegans & $b$ & 22 & $14: D$ & 28 & clear & none & 60 & C 33 SE SE SW & 4716040 & 582680 & 4100 \\
\hline $06 / 2495$ & 1026 & Thamnophis & elegans & 6 & 48 & $16: H$ & 28 & clear & none & 35 & EC 33 SE SE SW & 4716110 & 582680 & 4100 \\
\hline $06 / 26 / 95$ & 1855 & Thamnophis & elegans & $b$ & no & $15 \mathrm{C}$ & 28 & $\mathrm{Clo}$ & breezy & 25 & C 33 SE SE SW & 4716040 & 582675 & 4100 \\
\hline $06 / 28 / 95$ & 1849 & Thamnophis & elegans & $b$ & 57 & $16: B$ & 24 & clear & breery & 55 & TOSR 4 E SEC 33 SE SE SW & 4716110 & 582680 & 4100 \\
\hline $07 / 02 / 95$ & 1342 & Thamnophis & elegans & $b$ & no & & 29 & $\mathrm{M} / \mathrm{C}$ & breezy & 40 & TOS R4E SEC 33 NW NE NW & 4796580 & 582620 & 4100 \\
\hline $07 / 02 / 95$ & 1438 & Thamnophis & elegans & $\mathrm{b}$ & no & $16: E$ & 30 & cloudy & breezy & 45 & CC 33 SE SESW & 4716110 & 582680 & 4100 \\
\hline 07102295 & 1437 & Thermnophis & elegans & 6 & no & $16: B$ & 30 & clear & breezy & 30 & C 33 SE SE SW & 4716110 & 582880 & 4100 \\
\hline $07 / 102 / 95$ & 1446 & Thamnophis & elegans & $b$ & 65 & $15: 0$ & 29 & cloudy & breezy & 40 & TSSRAE & 4718060 & 582675 & 4100 \\
\hline $07104 / 95$ & 918 & Thamnophis & elepans & $\mathrm{b}$ & 71 & $17: B$ & 16 & clear & breezy & 40 & C 33 SW SE SW & 4718120 & 582620 & 4100 \\
\hline $07 / 104 / 95$ & 911 & Thamnophis & elecans & $\mathrm{b}$ & 70 & $16 \mathrm{~A}$ & 16 & clear & breezy & 50 & CC 33 SESE SW & $\frac{4110120}{4716110}$ & 582880 & $\frac{4100}{4100}$ \\
\hline $07 / 04 / 95$ & 904 & Thamnophis & elegans & $\mathrm{b}$ & 69 & $14: \mathrm{H}$ & 15 & clear & breezy & 60 & EC 33 SE SE SW & 4716040 & 582680 & 4100 \\
\hline $07 / 05 / 55$ & 1424 & Thamnophis & elecans & b & no & & 28 & Clear & breezy & 45 & EC $15 N E N E S W$ & 4702400 & 655530 & 5700 \\
\hline $07 / 05 / 95$ & 2130 & Thamnophis & eiegans & $b$ & no & $16 . \mathrm{C}$ & 23 & Clear & breagy & 35 & TOSRAE & 4716110 & 582680 & 4900 \\
\hline $07 / 05 / 95$ & 1254 & Thamnophis & elegans & $\mathrm{b}$ & no & & 27 & Clear & breezy & 30 & EC 15 NE NE NW & 4702520 & 655480 & 5700 \\
\hline $07105 / 95$ & 1241 & Thamnophis & elegans & 6 & no & & 26 & Clear & breezy & 30 & T11S R1E SEC 15 NE NE MW & 4702570 & 653560 & 5700 \\
\hline $07 / 08 / 95$ & $15<5$ & Thamnophis & eisgans & $i$ & no & & 32 & PIC & windy & 30 & TES RIE SEC 30 SW & 4727010 & 590010 & 6040 \\
\hline 0710845 & 1148 & Thamnophis & elegans & $b$ & no & $23: \mathrm{C}$ & 30 & clear & breezy & 30 & 33 SWINE SW & 4717780 & 5833380 & 4460 \\
\hline $07 / 0895$ & 1200 & Thamnophis & elegans & b & no & $22: 0$ & 31 & clear & breazy & 35 & 33 MW NE SW & 4716500 & 582550 & 4090 \\
\hline 895 & 1200 & Thamnophis & elegans & $\mathrm{b}$ & 73 & $22: 0$ & 31 & clear & breezy & 50 & $33 \mathrm{MWNESW}$ & 4716500 & 582550 & 4090 \\
\hline $07 / 0895$ & 1201 & Thamnophis & elegans & 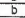 & 74 & $24: A$ & 32 & clear & breezy & 60 & C33 SWNE SW & 4717890 & 583360 & 4450 \\
\hline $07 / 108 / 95$ & 1218 & Thamnophis & elegans & 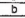 & no & $17: C$ & 32 & clear & breezy & 30 & TOS RAE SEC 33 SW SE SW & 4716120 & 582620 & 4100 \\
\hline $07 / 08 / 95$ & 1205 & Thamnoghis & eisgans & b & no & $23: \mathrm{H}$ & 32 & clear & breezy & 40 & T9S RAE SEC 33 SW NE SW & 4717780 & 583380 & 4460 \\
\hline $07 / 12 / 35$ & 1154 & Thamnophis & elegans & $b$ & 76 & $22 \mathrm{C}$ & 23 & clear & breezy & 50 & S3NWNESW & 4716500 & 582550 & 4090 \\
\hline $07 / 15 / 95$ & 11000 & Thamnophis & etegan 3 & $b$ & 82 & $16: \mathrm{A}$ & 22 & clear & none & 42 & 33 SE SE SW & 4716110 & 582680 & 4100 \\
\hline $07 / 15 / 95$ & 1005 & Thamnoghis & elepans & $b$ & $\frac{n_{2}}{n o}$ & 130 & $\frac{22}{21}$ & clear & none & 35 & TOSR4E & 4718090 & 582710 & 4180 \\
\hline $07 / 15 / 95$ & 945 & Thamnophis & eiegans & $b$ & 81 & $15: \mathrm{A}$ & 26 & clear & none & 50 & EC 33 SE SE SW & 4716040 & 582675 & 4100 \\
\hline $07 / 18 / 95$ & 1010 & Thamnophis & elegans & $b$ & 46 & $16: 8$ & 28 & $\mathrm{p} / \mathrm{c}$ & breezy & 51 & TOS RAE SEC 33 SE SE SW & 4716110 & 582680 & 4100 \\
\hline $07 / 18 / 95$ & 1030 & Thamnoohis & elegans & $b$ & no & $23: F$ & 31 & $p / c$ & ibreasy & 38 & C 33 SW NE SW & 7780 & 583330 & 4460 \\
\hline $07 / 1895$ & 945 & Thamnoghis & elepans & $\frac{5}{b}$ & 87 & $15: 8$ & 26 & $\frac{96}{9 / c}$ & breazy & $\frac{\infty}{43}$ & TSSRLE SEC 33 SE SESW & 4716020 & 582675 & 4100 \\
\hline $07 / 18 / 95$ & 950 & Thamnophis & elegans & $\mathrm{b}$ & 88 & $15: 0$ & 26 & $p / c$ & breezy & 39 & TOS RAE SEC 33 SE SE SW & 4716040 & 582675 & 4100 \\
\hline & 945 & Thamnophis & elegans & $b$ & 82 & $15: 8$ & 26 & $p / c$ & breezy & 42 & TSSRAE & 6040 & & 4100 \\
\hline $07 / 18 / 85$ & 1030 & Thamnoghis & elegans & $\mathrm{b}$ & no & $23: \mathrm{H}$ & 33 & $\mathrm{p} / \mathrm{c}$ & breezy & $\frac{14}{55}$ & EC 33 SWNE SW & 4717780 & 583380 & 4460 \\
\hline $07 / 18 / 95$ & 1040 & Themnophis & elegans & $b$ & no & $22 . \mathrm{C}$ & 27 & clear & breezy & 25 & EC 33 WWNE SW & 4796500 & 582550 & 4090 \\
\hline $07 / 21 / 95$ & 1858 & Thamnophis & elegans & b & 21 & $22: \mathrm{H}$ & 19 & cloudy & none & 45 & C 33 NWNE SW & 4716500 & 582550 & 4090 \\
\hline $07 / 21 / 55$ & 1924 & Thamnophis & elegans & $\mathrm{b}$ & 65 & $16: A$ & 20 & cloudy & none & 40 & TOS R4E SEC 33 SE SE SW & 4716110 & 582680 & 4100 \\
\hline $07 / 21 / 55$ & 1921 & Thamnophis & elegans & $\mathrm{b}$ & 46 & $16: C$ & 19 & cloudy & none & 51 & TOS RAE SEC 33 SE SE SW & 4718110 & 582680 & 4100 \\
\hline $07 / 21 / 95$ & 1849 & Thamnophis & elegans & $b$ & 76 & $23: G$ & 17 & cloudy & none & 50 & TOS RAE SEC 33 SW NE SW & 4797780 & 5833380 & 4460 \\
\hline $07 / 25 / 95$ & 955 & Thamnophis & elegans & $b$ & no & & 27 & clear & none & 50 & TSS R4E SEC $33 \mathrm{NW}$ SE SW & 4796450 & & 4100 \\
\hline $07 / 29 / 95$ & 740 & Thamnophis & elegans & $\mathrm{b}$ & no & $16: C$ & 23 & cloudy & breezy & 25 & TOSRAE SEC 33 SE & 4716110 & 582630 & 4100 \\
\hline $07 / 29 / 95$ & 748 & Thamnophis & elegans & $\mathrm{b}$ & no & 15.8 & 23 & cloudy & breezy & $\frac{52}{25}$ & TOS RAE SEC 33 SE & 4716040 & 582675 & 4100 \\
\hline $07 / 29 / 95$ & 730 & Thamnoohis & elegans & 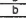 & no & $17: \mathrm{C}$ & 23 & cloudy & breezy & 40 & TSSRAE SEC 33 SW & $47: 6120$ & 582620 & 4100 \\
\hline $07 / 29 / 95$ & 748 & Thamnophis & elegans & b & 29 & 15.8 & 23 & cloudy & breezy & 45 & T9S RAE SEC 33 SE SE SW & 4716040 & 582675 & 4100 \\
\hline $07 / 29 / 95$ & 748 & Thamnophis & eiecans & b & no & $15 \mathrm{C}$ & 23 & cloudy & breezy & 30 & TOSRAES & 4716040 & & 4100 \\
\hline $07 / 29 / 95$ & 742 & Thamnophis & elegans & $\mathrm{b}$ & no & 16.4 & $\frac{53}{53}$ & cloudy & breezy & 45 & TOSRAES & 4716110 & 582630 & 4100 \\
\hline & 1851 & Thamnophis & elecans & $\vec{b}$ & 102 & $16: C$ & 25 & cleat & none & 30 & TSS RAE SEC 33 SE SE SW & 4718110 & 582630 & 4100 \\
\hline & 1003 & Thamnoohis & elegans & $\mathrm{b}$ & no & $16: C$ & 23 & cloudy & breezy & 45 & TOS RAE SEC 33 SE SE SW & 4716110 & 582630 & 4100 \\
\hline
\end{tabular}



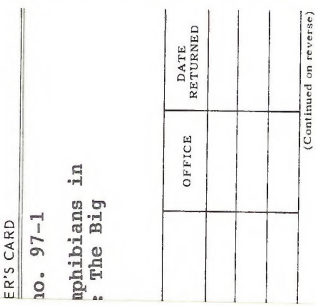

QL 84.2. IJ352 no. 97-1 88055389

Reptiles and amphibians in deep canyons : The Big

BLUU SU, O1-1SUA DENVER FEDERAL CENTER P.O. BOX 25047

DENVER, COLORADO 80225 